Automatic Track Initialization and Maintenance in

Heavy Clutter Using Integrated JPDA and

ML-PDA Algorithms 


\title{
AUTOMATIC TRACK INITIALIZATION AND MAINTENANCE IN HEAVY CLUTTER USING INTEGRATED JPDA AND ML-PDA ALGORITHMS
}

\author{
BY
}

K. HARISHAN, B.Sc. Eng.

\author{
A THESIS \\ SUBMitTED TO THE DEPARTMENT OF ELECTRICAL \& COMPUTER ENGINEERING \\ AND THE SCHOOL OF GRADUATE STUDIES \\ OF MCMASTER UNIVERSITY \\ IN PARTIAL FULFILMENT OF THE REQUIREMENTS \\ FOR THE DEGREE OF \\ Master of Applied Science \\ (c) Copyright by K. Harishan, March 2011 \\ All Rights Reserved
}


Master of Applied Science (2011)

(Electrical \& Computer Engineering)
McMaster University

Hamilton, Ontario, Canada

TITLE: Automatic Track Initialization and Maintenance in Heavy Clutter Using Integrated JPDA and ML-PDA Algorithms

AUTHOR: $\quad$ K. Harishan

B.Sc. Engineering, (Electrical Engineering)

University of Moratuwa, Sri Lanka

SUPERVISOR: $\quad$ Dr. T. Kirubarajan

NUMBER OF PAGES: $\mathrm{x}, 61$ 


\section{Abstract}

Target tracking in high clutter or low signal-to-noise environments presents many challenges to tracking systems. Joint Maximum Likelihood estimator combined with Probabilistic Data Association (JML-PDA) is a well-known parameter estimation solution for the initialization of tracks of very low observable and low signal-to-noiseratio targets in higher clutter environments. On the other hand, the Joint Probabilistic Data Association (JPDA) algorithm, which is commonly used for track maintenance, lacks automatic track initialization capability. This paper presents an algorithm to automatically initialize and maintain tracks using an integrated JPDA and JML-PDA approach that seamlessly shares information on existing tracks between the JML-PDA (used for initialization) and JPDA (used for maintenance) components. The motivation is to share information between the maintenance and initialization stages of the tracker, that are always on-going, so as to enable the tracking of an unknown number of targets using the JPDA approach in heavy clutter. The effectiveness of the new algorithm is demonstrated on a heavy clutter scenario and its performance is tested on negibouring targets with association ambiguity using angleonly measurements. 


\section{Acknowledgements}

I am especially grateful for the extensive and generous assistance of my supervisor, Professor T. Kirubarajan, who spent time with me each week to guide me through the thesis, provide funding and made numerous helpful suggestions to me.

I would like to thank Dr. R. Tharmarasa for his thorough reviews and perceptive comments, which greatly improved the quality of my thesis work. I would also like to thank Dr. S. Dumitrescu and Dr. J. Chen for being members of my committee. I appreciate all the time the members of my committee took to read this thesis, and for providing their input and thoughts on the subject. 


\title{
Notation and Abbreviations
}

\author{
Abbreviations \\ AI Amplitude Information \\ CJML-PDA Combined Joint Probabilistic Data Association \\ CRLB Cramer-Rao Lower Bound \\ FIM Fisher Information Matrix \\ IMM Iterative Multiple Model \\ IRF Information Reduction Factor \\ JLLR Joint Log Likelihood Ratio \\ JML-PDA Joint Maximum Likelihood Probabilistic Data Association \\ JPDAF Joint Probabilistic Data Association Filter \\ LLR Log Likelihood Ratio \\ ML-PDA Maximum Likelihood Probabilistic Data Association \\ MHT Multiple Hypothesis Tracker \\ PHD Probabilistic Hypothesis Density \\ RMSE Root Mean Squared Error \\ SNR Signal-to-Noise Ratio \\ TBD Track-Before-Detect
}




\section{Notations}

$V \quad$ Volume of surveillance region (measurement space)

$N \quad$ Number of existing targets

$N^{*} \quad$ Number of new targets

$\xi \quad$ Position

$\dot{\xi} \quad$ Velocity

$\eta \quad$ Altitude

$\gamma \quad$ Gating threshold

$\mathbf{E}[\cdot] \quad$ Expected value

$\mathbf{f}(\cdot) \quad$ Target/sensor motion model

$\mathbf{F}(\cdot) \quad$ Linearized plant matrix

$\mathbf{h}(\cdot) \quad$ Nonlinear measurement model

$\mathbf{H}(\cdot)$ Linearized $H$

$H_{1} \quad$ Target-present hypothesis

$H_{0} \quad$ No target-present hypothesis

$\tau \quad$ Detection threshold

$\pi_{m} \quad$ Miss probability

$i \quad$ Discrete time step

T Sampling time

$i_{0} \quad$ Reference time (discrete)

$i_{k} \quad$ Current time

$N_{w} \quad$ Length of a window (discrete)

$\Phi(\cdot) \quad$ Log-likelihood ratio

$\Lambda(\cdot) \quad$ Likelihood ratio

$\mu(\cdot) \quad$ Possion distribution

$a_{j}(\cdot)$ Amplitude measurement 
$\Upsilon \quad$ Track validation test statistic

$\lambda \quad$ Possion expected value

$P_{d}^{t} \quad$ Probability of detection of target $t$

$P_{f a} \quad$ Probability of false alarm

d Signal-to-noise ratio

$\sigma_{\theta} \quad$ Sensor noise standard deviation (angle-only)

$\sigma_{t} \quad$ Target process noise standard deviation

$\beta_{j}^{t}(i) \quad$ Marginal association probability ( $j$ th measurement, $t$ th target, $i$ th time)

$\mathcal{N}[\mu, \sigma] \quad$ Normal distribution with mean $\mu$ and standard deviation $\sigma$

$\mathcal{U}[a, b] \quad$ Uniformly distributed in $[a, b]$

$\mathcal{Q}(\cdot) \quad$ Information reduction factor

$J \quad$ Fisher information matrix 


\section{Contents}

Abstract

Acknowledgements $\quad$ iv

Notation and Abbreviations $\quad$ v

1 Introduction 1

1.1 Introduction . . . . . . . . . . . . . . . . . . . 1

1.2 Motivation and Contribution of the Thesis . . . . . . . . . . . . . 4

1.3 Organization of the Thesis . . . . . . . . . . . . 5

1.4 Related Publications ... . . . . . . . . . . . . . . 6

2 Problem Formulation $\quad 7$

2.1 Dynamic Models . . . . . . . . . . . . . . . . . . . . . 7

2.2 Association Events . . . . . . . . . . . . . . . . . . . . . . 11

2.3 ML-PDA Tracker . . . . . . . . . . . . . . . . . . . . . . . . . 14

2.4 JML-PDA Tracker . . . . . . . . . . . . . . . . . . . . . . . . 18

2.5 JPDA Tracker . . . . . . . . . . . . . . . . . . . . . . . . . . . . . . . 19 
3 Combined JML-PDA and JPDA Algorithms

3.1 JML-PDA Algorithm: $N$ targets . . . . . . . . . . . . . . . . . . 23

3.2 C.JML-PDA Algorithms . . . . . . . . . . . . . . . . . . 28

3.3 Log-Likelihood Ratio Optimization . . . . . . . . . . . . . . . . 33

3.4 Track Validation . . . . . . . . . . . . . . . . . . . . . 34

3.5 Measurement Gating . . . . . . . . . . . . . . . . . . . . . . 37

3.6 CRLB and Covariance of the Estimates . . . . . . . . . . . . . . . 38

3.6.1 Information Reduction Factor : 1D Measurement Space . . . . 40

4 Simulation Studies $\quad 47$

4.1 Test Scenario . . . . . . . . . . . . . . . . . . . . . 47

4.2 Restricted Direct Subspace Search (RDSS) . . . . . . . . . . . 50

$5 \quad$ Numerical Results $\quad 52$

6 Conclusions and Future Work $\quad 57$

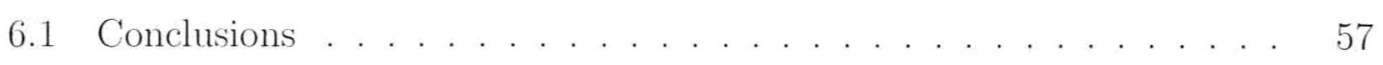

6.2 Future Work . . . . . . . . . . . . . . . . . 58 


\section{List of Figures}

2.1 Validation gate . . . . . . . . . . . . . . . . . . 12

$3.12 \mathrm{D}$ view of $4 \mathrm{D}$ log-likelihood-ratio with known velocities and angle-only measurements. . . . . . . . . . . . . . . . . . . . 35

4.1 Target and sensor motion ( Y. Bar-Shalom, X. R. Li and T. Kirubarajan, 2001, pg. 388) . . . . . . . . . . . . . . . . 48

5.1 Target and sensor trajectories. . . . . . . . . . . . . . . . 52

5.2 Estimated trajectories of target 2 over 100 runs. . . . . . . . . . 54

5.3 Estimated velocities of targets 1 and 2 over 100 runs. . . . . . . . 55

5.4 RMSE in position estimates of target states. . . . . . . . . . . 56

5.5 RMSE in velocity estimates of target states. . . . . . . . . . . . 56 


\section{Chapter 1}

\section{Introduction}

\subsection{Introduction}

Track Very Low Observable (VLO) targets in high clutter presents many difficulties in target tracking. The term "VLO targets" is also known as targets with low Signal-toNoise Ratio (SNR). One challenge is that choosing a threshold to have desirable target detection probability and false alarm probability. A better target detection probability requires a lower threshold. However, lowering the threshold has the opposite impact on on probability of false alarm. In addition, as the number of false alarms increases Kalman filter based algorithms rapidly lose efficiency and effectiveness. Thus, new approaches have been introduced for VLO target tracking. Track-Before Detect (TBD) is one such technique that is useful when the SNR is low (C. Jauffret and Y. Bar-Shalom, 1990), (T. Kirubarajan and Y. Bar-Shalom, 1996), (L. A. Johnston and V. Krishnamurthy, 2002). TBD performs track estimation and track acceptance simultaneously without applying any threshold or lower threshold sensor data. TBD algorithms typically operate on data over several scans as a batch. Therefore, TBD 
algorithms are better solutions to initiate or sustain a track (W. Blanding, P. Willett and Y. Bar-Shalom, 2007a). However, the computational complexity of TBD algorithms are generally much higher than that of conventional trackers like Kalman filters.

One TBD that has been used in many practical system is the Maximum Likelihood Probabilistic Data Association (ML-PDA) estimator. MLPDA was first introduced in (C. Jauffret and Y. Bar-Shalom, 1990) to estimate single target parameter using a batch of bearing and frequency measurements in very high clutter or SNR environment. The ML-PDA tracker uses data over a batch of measurements to compute track estimates using a sliding window. This is an effective approach to initialize tracks in high clutter, but it assumes a deterministic target models (no target process noise). The algorithm formulates a Log-Likelihood Ratio (LLR) from a set of sensor data, and then the track estimate is given by target state that maximizes the LLR. The use of the additional Amplitude Information (AI), a measurement feature, in ML-PDA in (T. Kirubarajan and Y. Bar-Shalom, 1996) facilitated the acquisition of even weaker targets. Furthermore, in (T. Kirubarajan, Y. Bar-Shalom and D. Lerro, 2001), ML-PDA was used to provide consistent initialization, whose initial estimate and covariance were given to an interacting multiple model probabilistic data association filter with amplitude information (IMM-PDAF-AI) for maintenance. This was also demonstrated on an angle-only tracking problem in clutter. Note that the fundamental assumption in these approaches is that there is only one target. In addition, the initialization and maintainance phases were independent except for passing the state from one stage to the next. 
An adaptive sliding window in ML-PDA was used in (M. R. Chummun, Y. BarShalom and T. Kirubarajan, 2002) to detect a fast-moving and possibly maneuvering target using an imaging sensor. This resulted in better detection capability than an Interacting Multiple Model estimator combined with Multiple Hypothesis Tracking (IMM-MHT) in high clutter environments. Note that the MHT algorithm is inherently capable of handling multiple targets (X. R. Li and V. P. Jilkov, 2003), (D. Lerro and Y. Bar-Shalom, 2002).

A multitarget ML-PDA algorithm known as the Joint ML-PDA algorithm (JMLPDA) was used in (W. Blanding, P. Willett and Y. Bar-Shalom, 2007a), (W. Blanding, P. Willett and Y. Bar-Shalom, 2007b) for simultaneous track maintenance. This approach is an extension of the standard ML-PDA technique, but here the LLR is formed as a combination of state vectors in order to simultaneously estimate the confirmed track estimates. For detection, the measurements associated with the conformed tracks were removed from each frame of data and the optimization was done with those measurements with ML-PDA. The process was repeated until no more targets were found in a window. This assumed that new targets are well separated in the measurement space. However, this JML-PDA implementation lacks track initialization capability of neighboring targets.

In presence of clutter, another effective approach to data association issue is a Probabilistic Data Association (PDA) tracker, a single target tracker in clutter (Y. Bar-Shalom and X. R. Li, 1995). The PDA framework calculates a probability for each measurement being target originated. Then, measurement-to-track association handled softly with those probabilities among all possible measurements. A multitarget version, the Joint Probabilistic Data Association (JPDA) approach is same 
as PDA approach, except that, probabilities are computed as joint probabilities to account for the fact that measurements can associate to more than one target. The JPDA algorithm has been reported in (Y. Bar-Shalom and X. R. Li, 1995), (A. Rodningsdy, Y. Bar-Shalom, O. Hallingstad and J. Glattetre, 2007), (Y. Bar-Shalom, F. Daum and J. Huang, 2009) to be suitable for tracking of multiple targets in presence of high clutter. However, the assumption in these algorithms is that the number of targets in the surveillance region is known. The main motivation of JPDA/PDA algorithm is that for track maintenance.

\subsection{Motivation and Contribution of the Thesis}

In this thesis, a new way of initializing and maintaining tracks for multiple targets within the PDA framework through the seamless integration of the JML-PDA and the JPDA is presented. The motivation is to develop a multitarget tracker capable of handling the initiation and maintenance of low observable tracks in high clutter environments. The novelty of this Combined JML-PDA (CJML-PDA) and JPDA tracker is in the sharing of information between the two tracking modules, which in previous batch-recursive approaches were treated as two independent (and consecutive) stages of tracking. Past research assumed that the new targets are well separated in the measurement space (W. Blanding, P. Willett and Y. Bar-Shalom, 2007a). The ML-PDA is repeated on residual measurement set for single target initiation and JML-PDA is used for track maintenance of possible neighbouring targets.

As the main contribution of this thesis, the JML-PDA algorithm is extended to estimate any number of new targets' estimates with the aid of conformed track information in JPDA. With the presence of many targets in high clutter, tracking 
with JML-PDA requires a higher computation time to calculate new and confirmed targets (detection and maintenance) estimates. Also, the processing time in JMLPDA exponentially increasing as the numbers of targets and measurements increase. However, an advantage of the JML-PDA algorithm is that have been shown to be powerful for tracking single and multiple targets in high clutter. Consequently, a new version of the JML-PDA algorithm called the CJML-PDA, for track initialization with JPDA is presented in this thesis. The advantage of JPDA is that it can handle high clutter and miss detections by considering all possible data association between targets and measurements and meantime, the JPDA tracker does not require an exponentially increasing amount of processor times as in JML-PDA.

\subsection{Organization of the Thesis}

The thesis is organized as follows. Chapter 2 starts with symbols and notations useful in reporting, and follows with a brief introduction of ML-PDA, JML-PDA and JPDA algorithms. Chapter 3.2 presents the formulation of the new algorithm, including track validation, the Cramer-Rao Lower Bound (CRLB) for the estimator and measurement gating. The calculation of the CRLB, which quantifies the accuracies achievable by any estimator, requires an information reduction factor that accounts for the loss of information due to false alarms, missed detections and the presence of multiple targets. The information reduction factor considers not only non-zero false alarm probability and non-unity detection probability, but also the inter-target distance is presented in Section 3.2. Simulation results validing the theory are given in Section 5. 


\subsection{Related Publications}

- K. Harishan, R. Tharmarasa, T. Kirubarajan and T. Thayaparan, "Automatic track initialization and maintenance in heavy clutter using integrated JPDA and ML-PDA algorithms, Proceedings of the Signal and Data Processing of Small Targets, San Diego, CA, August 2011. 


\section{Chapter 2}

\section{Problem Formulation}

This chapter consists of the necessary notations for the tracker implementation which includes the kinematic model and measurement models. A brief summary of MLPDA, multitarget ML-PDA (JML-PDA), and JPDA algorithms are also presented in this chapter.

\subsection{Dynamic Models}

The $t^{\text {th }}$ target's state at any discrete time $i$ is defined by

$$
x^{t}(i+1)=\mathbf{f}^{t}\left(x^{t}(i), T\right)+\nu^{t}(i)
$$

where $\mathbf{f}(\cdot)$ is the target's motion model, possibly a nonlinear function of target state at current time $i$ and sampling interval $T$. In the above $\nu^{t}(i)$ is known as the target's 
process noise, and, assuming zero mean Gaussian, its variance is given by

$$
Q^{t}(i)=\mathbf{E}\left[\nu^{t}(i) \nu^{t}(i)^{\prime}\right]
$$

Linearized model of the target motion model can be found using the Taylor expansion of $\mathrm{f}^{t}\left(x^{t}(i), T\right)$ (Y. Bar-Shalom, X. R. Li and T. Kirubarajan, 2001) as

$$
\mathbf{F}^{t}\left(x^{t}(i), T\right)=\left.\left(\frac{\partial \mathrm{f}^{t}\left(x^{t}(i), T\right)}{\partial x^{t}(i)}\right)\right|_{x^{t}(i)=\hat{x}^{t}(i)}
$$

To ensure observability criteria, bearings-only tracking requires a sufficient condition on sensor motion (T. Kirubarajan and Y. Bar-Shalom, 1996). State of the sensor platform at discrete time $i$ is defined by

$$
y(i+1)=\mathrm{f}^{s}(y(i), T)+\nu^{s}(i)
$$

As in the target case, $\mathrm{f}^{\mathrm{s}}(\cdot)$ could be a nonlinear function of sensor state at current time $i$ and sampling interval $T$. It is assumed that sensor position is known perfectly.

Now, the relative state of a target from the sensor, can be written as

$$
x^{t s}(i)=x^{t}(i)-y(i)
$$

Window based JML-PDA and ML-PDA algorithms require a data measurement set of $N_{W}$ frames of recent data (W. Blanding, P. Willett and Y. Bar-Shalom, 2007a). Let $Z^{N_{w}}$ be a batch of measurements from time $i_{0}$ to $i_{k}$, the reference time and current 
time, respectively. Such a batch is given by

$$
Z^{N_{w}}=\left\{Z\left(i_{0}\right), Z\left(i_{0}+1\right), \ldots, Z(i), \ldots, Z\left(i_{k}\right)\right\}
$$

where $Z(i)$ is the set of all measurements at time $i$, and it is given by,

$$
Z(i)=\left\{z_{1}(i), z_{2}(i), \ldots, z_{j}(k), \ldots, z_{m_{i}}(i)\right\}
$$

where $m_{i}$ is the number of measurements at time $t_{i}$. Also,

$$
N_{w}=i_{k}-i_{0}+1
$$

Depending on the origin of measurement $z_{j}(i)$, it is given by

$$
z_{j}(i)= \begin{cases}\mathbf{h}\left(x^{t s}(i), i\right)+w(i) & \text { target-originated } \\ v(i) & \text { false alarm }\end{cases}
$$

where $\mathbf{h}(\cdot)$ is the measurement model, which is a function of target state, sensor state and current time $i$. Measurement noise $w(i)$ is independent of target process noise and assumed to be white Gaussian with zero means and with variance

$$
R(i)=\mathbf{E}\left[w(i) w(i)^{\prime}\right]
$$

In the above, $v(i)$ is assumed to be a uniformly distributed random variable across the surveillance region's volume $V$.

A linearized version of the measurement model can be obtained using the Taylor 
expansion of $\mathbf{h}\left(x^{t s}(i), i\right)$, same as

$$
\mathbf{H}\left(x^{t s}(i), i\right)=\left.\left(\frac{\partial \mathbf{h}\left(x^{t s}(i), i\right)}{\partial x}\right)\right|_{x=\hat{x}^{t s}(i)}
$$

Amplitude Information (AI) can be included in the measurement to increase the observability of targets. The amplitude measures the intensity of the signal at the output of the signal processor. Here, the AI is used to validate the measurement with a threshold. The output signal is usually Rayleigh distributed, (T. Kirubarajan and Y. Bar-Shalom, 1996). The probability density function of amplitude measure a due to noise only measurement (false alarm) can written as

$$
p_{0}(a)=a \exp \left(-\frac{a^{2}}{2}\right)
$$

The signal generated from a target is given by

$$
p_{1}(a)=\frac{a}{1+d} \exp \left(-\frac{a^{2}}{2(1+d)}\right)
$$

where $d$ is the value of target SNR.

A threshold $\tau$ is chosen to declare the probability of detection and is given by

$$
P_{d}=\int_{\tau}^{\infty} p_{1}(a) d a
$$

Similarly, the probability of a false measurement can written as

$$
P_{f a}=\int_{\tau}^{\infty} p_{0}(a) d a
$$


It is clear that the probability of detection and probability of false alarm will increase with a decreasing threshold. A suitable $\tau$ is necessary for a given SNR to satisfy certain level of $P_{d}$ and $P_{f a}$. With that threshold, the validated measurements probability density function due to noise only and target generated, $p_{0}^{\tau}(a)$ and $p_{1}^{\tau}(a)$, respectively, are given by

$$
\begin{gathered}
p_{0}^{\tau}(a)=\frac{1}{P_{f a}} p_{0}(a)=\frac{1}{P_{f a}} a \exp \left(-\frac{a^{2}}{2}\right) \\
p_{0}^{\tau}(a)=\frac{1}{P_{f a}} p_{1}(a)=\frac{a}{P_{d}(1+d)} \exp \left(-\frac{a^{2}}{2(1+d)}\right)
\end{gathered}
$$

Then, $P_{d}$ and $P_{f a}$ satisfy

$$
\begin{aligned}
P_{d} & =\exp \left(-\frac{\tau^{2}}{2(1+d)}\right) \\
P_{f a} & =\exp \left(-\frac{\tau^{2}}{2}\right)
\end{aligned}
$$

\subsection{Association Events}

The PDA approach uses all possible joint association events corresponding to measurementto-target association possibilities and track is computed with all preservations. For the process of derivation, introduce a variable called "association vector"

$$
E(i)=\left[e_{1}(i), e_{2}(i), \ldots, e_{m_{i}}(i)\right]^{\prime}
$$

Where event $E(i)$ indicates which measurement originated from which target. For 
example, If $e_{j}(i)=t$, it indicates that the $j$ th measurement is originated from the th tharget. If $e_{j}(i)$ equals zero is a false alarm.

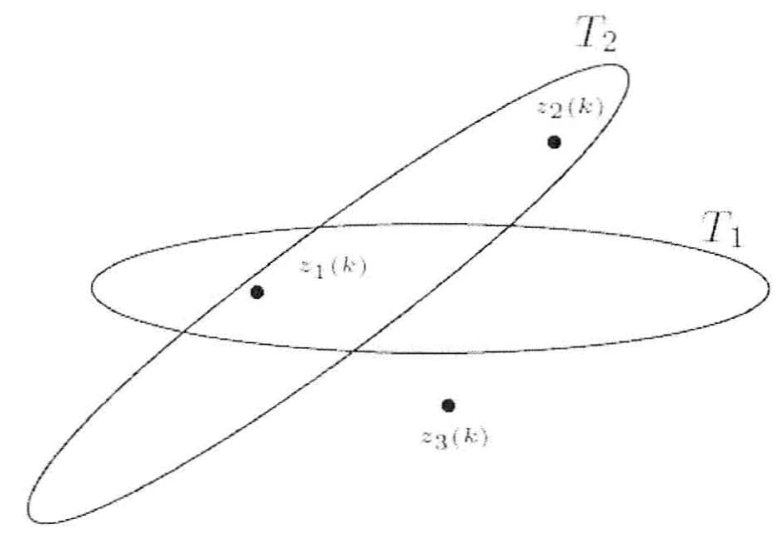

Figure 2.1: Validation gate

Neighbouring targets in heavy clutter generate many association vectors, and all of them should be accounted for data association. For instance, consider the multiple validations in Figure 2.1. It shows two target gates and three measurements. It is clear that measurement 1 is common to both gates. This measurement can be associated with either targets, but the assumption in this paper is that at most one measurement from each target and vise versa. Measurement 2 is only in the second validation gate. The measurement 3 is not considered to have come from any target. 
With these facts, the set of all possible association events can written as

$$
\mathbf{E}(i)=\left\{\begin{array}{c}
(0,0) \\
(1,0) \\
(2,0) \\
(0,2) \\
(1,2)
\end{array}\right\}
$$

This indicates which targets are detected in an association event. Element $d_{t}(i) \triangleq$ $D(E(i), t)$ is one if the $t$ th target is detected in the association event. Note that this does not include track initialization.

Now, define a detection vector of an associate event, given by

$$
D(E(i))=\left[d_{1}(i), d_{2}(i), \ldots, d_{N}(i)\right]
$$

Thus, the total number of detections in an event $E(i)$, i.e $d(D(E(i)))$, can be evaluated as

$$
d(D(E(i)))=\sum_{t=1}^{N} D(E(i), t)
$$

Again, consider the example given in (2.20). The detection vector for each given 
association events can be written as

$$
D(\mathbf{E}(i))=\left\{\begin{array}{c}
(0,0) \\
(1,0) \\
(0,1) \\
(0,1) \\
(1,1)
\end{array}\right\}
$$

\subsection{ML-PDA Tracker}

A detailed derivation of the ML-PDA algorithm incorporating amplitude information can be found in (T. Kirubarajan and Y. Bar-Shalom, 1996). Summery of a generalized ML-PDA version is presented this section.

Usually, the ML-PDA algorithm uses the following assumptions:

- Only single target is present in each data frame with a given detection probability $P_{d}$, and detections are independent across frames

- At most one measurement per frame corresponds to the target

- The target operates according to deterministic kinematics with no process noise. The target's kinematic state at a given reference time $i_{0}$ is related to the target's state at anytime. Therefore, equation (2.1) can be rewritten as

$$
x(i)=\mathrm{f}\left(x\left(i_{0}\right), i\right)
$$

- False detections are distributed uniformly in the search volume $V$ 
- The number of false detections is Poisson distributed with a known expected number of false measurements $\lambda$ in the volume $V$ with probability mass function being given by

$$
\mu_{f a}\left(m_{i}\right)=\frac{\exp (-\lambda V)(\lambda V)^{m_{i}}}{m_{i} !}
$$

- Amplitude of the target originated and false detections are distributed according to Reyleigh probability density function $p_{1}(a)$ and $p_{0}(a)$, respectively. The target SNR, which affects $p_{1}(a)$, is either known or estimated in real time.

- Target-originated measurements are corrupted with additive zero-mean white Gaussian noise

- Measurements obtained at different times are conditionally independent, i.e.,

$$
P\left(Z\left(i_{1}\right), Z\left(i_{2}\right) \mid x\right)=P\left(Z\left(i_{1}\right) \mid x\right) P\left(Z\left(i_{2}\right) \mid x\right)
$$

The probability density function of target-originated measurement given the target state using ( 2.9 ) can be written as

$$
p\left(z_{j}(i) \mid x\left(i_{0}\right)\right)=\mathcal{N}\left[z_{j}(i) ; \mathbf{h}\left(x^{t}\left(i_{0}\right), y(i), i\right), R(i)\right]
$$


Expanded version of the above function is given by

$$
\begin{aligned}
& p\left(z_{j}(i) \mid x\left(i_{0}\right)\right)= \\
& \frac{1}{(2 \pi)^{\frac{k}{2}}|R(i)|^{\frac{1}{2}}} \exp \left(-\frac{1}{2}\left[z_{j}^{s}(i)-\mathbf{h}\left(x\left(i_{0}\right), y(i), i\right)\right] R(i)^{-1}\left[z_{j}^{s}(i)-\mathbf{h}\left(x\left(i_{0}\right), y(i), i\right)\right]^{\prime}\right)
\end{aligned}
$$

where $k$ is the dimension of the measurement space.

Having the above ML-PDA assumptions and definitions, the likelihood function $P(Z(i) \mid x(i))$ of a single frame of data is formed as weighted sum of all possible target detection events, that is,

$$
P(Z(i) \mid x(i))=\left(1-P_{d}\right) \frac{\mu\left(m_{i}\right)}{V^{m_{i}}}+P_{d} \frac{\mu\left(m_{i}-1\right)}{V^{m_{i}-1} m_{i}} \sum_{j=1}^{m_{i}} p\left(z_{j}(i) \mid x\left(i_{0}\right)\right)
$$

The likelihood function over $N_{w}$ frames is the product of single frame likelihood functions. Thus, it can be given as

$$
\begin{aligned}
P\left(Z^{N_{w}} \mid x\left(i_{0}\right)\right) & =\prod_{i=i_{0}}^{i_{k}} P(Z(i) \mid x(i)) \\
& =\prod_{i=i_{0}}^{i_{k}}\left[\left(1-P_{d}\right) \frac{\mu\left(m_{i}\right)}{V^{m_{i}}}+P_{d} \frac{\mu\left(m_{i}-1\right)}{V^{m_{i}-1} m_{i}} \sum_{j=1}^{m_{i}} p\left(z_{j}(i) \mid x\left(i_{0}\right)\right)\right]
\end{aligned}
$$

Dividing ( 2.30 ) by the likelihood given all measurements are false detections which is given by

$$
\prod_{i=i_{0}}^{i_{k}} \frac{\mu\left(m_{i}\right)}{V^{m_{i}}}
$$


and taking the logarithm of the resulting function results in a dimensionless and more compact form, given by

$$
\Phi\left[Z^{N_{w}}, x\left(i_{0}\right)\right]=\sum_{i=i_{0}}^{i_{k}} \ln \left[\left(1-P_{d}\right)+\frac{P_{d}}{\lambda} \sum_{j=1}^{m_{i}} p\left(z_{j}(i) \mid x\left(i_{0}\right)\right)\right]
$$

The function is known as the maximum likelihood probabilistic data association log likelihood ratio (ML-PDA LLR) (W. Blanding, P. Willett and Y. Bar-Shalom, 2007b). The maximum likelihood approach finds the target parameter $\hat{x}\left(i_{0}\right)$ that maximizes log likelihood ratio function. That is,

$$
\hat{x}\left(i_{0}\right)=\underset{x\left(i_{0}\right)}{\arg \max } \Phi\left(Z^{N_{w}}, x\left(i_{0}\right)\right)
$$

When amplitude information is incorporated ( 2.34 ) can be rewritten as Kirubarajan and Y. Bar-Shalom, 1996)

$$
\Phi\left[[Z, a]^{N_{w}}, x(i)\right]=\sum_{i=i_{0}}^{i_{k}} \ln \left[\left(1-P_{d}\right)+\frac{P_{d}}{\lambda} \sum_{j=1}^{m_{i}} \rho_{j}(i) p\left(z_{j}(i) \mid x\left(i_{0}\right)\right)\right]
$$

where $\rho_{j}(i)$ is the amplitude ratio of the $j$ th measurement is given by

$$
\begin{aligned}
\rho_{j}(i) & =\frac{p_{1}^{\tau}\left(a_{j}(i)\right)}{p_{0}^{\tau}\left(a_{j}(i)\right)} \\
& =\frac{P_{f a}}{P_{d}(1+d)} \exp \left(a_{j}(i) \frac{d}{1+d}\right)
\end{aligned}
$$




\subsection{JML-PDA Tracker}

This section consists of a detailed derivation of the JML-PDA for $N$ number of targets. A detail derivation of the JML-PDA algorithm incorporating amplitude information can be found in (W. Blanding, P. Willett and Y. Bar-Shalom, 2007a). This was given only for two target scenarios, but JML-PDA can be formulated for any number of targets. The JMLPDA uses the assumptions in the ML-PDA, but the multitarget version makes the following additional assumptions:

- $N$ conformed targets are present in each data frame with a given detection probability, and detections are independent across frames

- At most one measurement per frame corresponds to each target

- A measurement cannot be associated with more than one target

- Measurements originating from different targets are independent

- Target originated measurement errors have the same distribution for all targets, that is, they are a function of the sensor, and not the target.

The formulation the JLLR to jointly estimates track estimates of the two targets can be found in (W. Blanding, P. Willett and Y. Bar-Shalom, 2007b) and (W. Blanding, P. Willett and Y. Bar-Shalom, 2007a). JML-PDA Joint LLR (JLLR) of 
$N_{W}$ frames can be found as

$$
\begin{gathered}
\Phi\left[[Z, a]^{N_{w}},\left[x^{1}\left(i_{0}\right), x^{2}\left(i_{0}\right)\right]\right]=\sum_{i=i_{0}}^{i_{k}} \ln \left[\left(1-P_{d}^{1}\right)\left(1-P_{d}^{2}\right)\right. \\
+\frac{P_{d}^{1}\left(1-P_{d}^{2}\right)}{\lambda} \sum_{j=1}^{m_{i}} \rho_{j}(i) p\left(z_{j}(i) \mid x^{1}(i)\right)+\frac{P_{d}^{2}\left(1-P_{d}^{1}\right)}{\lambda} \sum_{j=1}^{m_{i}} \rho_{j}(i) p\left(z_{j}(i) \mid x^{2}(i)\right) \\
\left.+\quad \frac{P_{d}^{1} P_{d}^{2}}{\lambda^{2}} \sum_{j=1}^{m_{i}} \sum_{\substack{l=1 \\
l \neq j}}^{m_{i}} p\left(z_{j}(i) \mid x^{1}(i)\right) p\left(z_{j}(i) \mid x^{2}(i)\right)\right]
\end{gathered}
$$

The extension of above JLLR to an arbitrary number of targets is given in Section 3.1. This includes all possible target detection events given measurements and number of targets.

\subsection{JPDA Tracker}

In this section, the core equations in the JPDA tracker is presented. The detailed derivation of this algorithm can be found in (Y. Bar-Shalom and X. R. Li, 1995). The JPDA is used for to maintain existing tracks with no buitin capability to initiate new tracks. Here, the parametric version of JPDA is used since the assumption is that false alarms are Possion distributed. The nonparametric version does not require prior knowledge of the spatial density of the false measurements, a realistic method where the false measurements (D. Lerro and Y. Bar-Shalom, 1993).

Generally, the JPDA algorithms makes the following assumptions:

- $N$ established targets are present at any time $i$, and the target state is estimated as $\hat{x}^{t}(i \mid i)$ with associate covariance $\hat{P}^{t}(i \mid i)$

- The past information about $t$ th target is summarized approximately by the 
Gaussian distribution, i.e.,

$$
p\left(x^{t}(i) \mid Z^{i-1}\right)=\mathcal{N}\left[x^{t}(i) ; \hat{x}^{t}(i \mid i-1), P^{t}(i \mid i-1)\right]
$$

- The number of false detections is Poisson distributed as in JML-PDA/ML-PDA

The marginal association probability of $j$ th measurement being associated to $t$ th target can be written as

$$
\beta_{j}^{t}(i)=\sum_{\forall E_{j}^{t}(i)} P\left(E_{j}^{t}(i) \mid Z^{i}\right)
$$

The term $P\left(E_{j}^{t}(i) \mid Z^{i}\right)$ is the probability of an individual event is given by

$$
\begin{aligned}
P\left(E_{j}^{t}(i) \mid Z^{i}\right)= & \frac{1}{C} \times \frac{\left(m_{i}-d(D(E(i)))\right) !}{m_{i} !} \mu_{F A}\left(m_{i}-d(D(E(i)))\right) \\
& \cdot \prod_{t=1}^{N}\left(P_{d}^{t}\right)^{D(E(i), t)}\left(1-P_{d}^{t}\right)^{(1-D(E(i), t))} \prod_{j=1}^{m_{i}} p\left(z_{j}(i) \mid x^{e_{j}(i)}(i)\right)
\end{aligned}
$$

where $C$ is the normalizing constant and $E_{j}^{t}(i)$ is the association event that $j$ th measurement associated to th target.

The $t$ th target JPDA estimate at time $i$ can be written as

$$
x^{t}(i \mid i)=\sum_{j=1}^{m_{i}} \beta_{j}^{t}(i) \hat{x}^{t j}(i \mid i)
$$

where $\hat{x}^{t j}(i \mid i)$ is the updated state estimates conditioned on the event that the $j$ th measurement is correct, and $\beta_{j}^{t}$ is the probability of event $j$. Thus, $\hat{x}^{t j}(i \mid i)$ is given 
by

$$
\hat{x}^{t j}(i \mid i-1)=\hat{x}^{t}(i \mid i-1)+W^{t}(i) \nu_{j}^{t}(i)
$$

where $\hat{x}^{t}(i \mid i-1)$ is the predicted estimates of the state at time $i, \nu_{j}^{t}(i)$ is the innovation associated with the $j$ th measurement and $W^{t}(i)$ is the Kalman filter gain, (Y. BarShalom and X. R. Li, 1995).

The innovation can be written as

$$
\nu_{j}^{t}(i)=z_{j}(i)-\hat{z}^{t}(i \mid i-1)
$$

where $\hat{z}^{t}(i \mid i-1)$ is the predicted measurement of the $t$ th target and is given by

$$
\hat{z}^{t}(i \mid i-1)=\mathbf{h}\left(\hat{x}^{t}(i \mid i-1), y(i), i\right)
$$

The gain is given by

$$
W_{j}^{t}(i)=P^{t}(i \mid i-1)\left(\mathbf{H}\left(\hat{x}^{t}(i \mid i-1), y(i), i\right)\right)^{\prime}\left(S(i)^{t}\right)^{-1}
$$

where $P^{t}(i \mid i-1)$ is the predicted target state associated covariance given by

$$
P^{t}(i \mid i-1)=\left(\mathbf{F}\left(\hat{x}^{t}(i \mid i-1), i\right)\right) P^{t}(i-1 \mid i-1)\left(\mathbf{F}\left(\hat{x}^{t}(i \mid i-1), i\right)\right)^{\prime}+Q(i)
$$

and $S(i)^{t}$ is the innovation covariance given by

$$
S(i)^{t}=\left(\mathbf{H}\left(\hat{x}^{t}(i \mid i-1), y(i), i\right)\right) P^{t}(i \mid i-1)\left(\mathbf{H}\left(\hat{x}^{t}(i \mid i-1), y(i), i\right)\right)^{\prime}+R^{t}(i)
$$


The state estimation covariance is updated as

$$
\begin{aligned}
P^{t}(i \mid i) & =\beta_{0}^{t}(i) P^{t}(i \mid i-1)+\left(1-\beta_{0}^{t}(i)\right)\left(P^{t}(i \mid i-1)-W^{t}(i)\left(S(i)^{t}\right)^{-1}\left(W^{t}(i)\right)^{\prime}\right) \\
& +W^{t}(i)\left[\sum_{\forall j} \beta_{j}^{t}(i) \nu_{j}^{t}(i)\left(\nu_{j}^{t}(i)\right)^{\prime}-\left(\sum_{\forall j} \beta_{j}^{t}(i) \nu_{j}^{t}(i)\right)\left(\sum_{\forall j} \beta_{j}^{t}(i) \nu_{j}^{t}(i)\right)^{\prime}\right]
\end{aligned}
$$




\section{Chapter 3}

\section{Combined JML-PDA and JPDA}

\section{Algorithms}

\subsection{JML-PDA Algorithm: $N$ targets}

In this section a JML-PDA JLLR formulation for arbitrary number of targets is presented. The assumptions from Section 2.4 are kept for any number of targets as well. Thus, the joint likelihood function of $N$ targets, which is the probability density function of measurements in $Z(i)$ conditioned on all target states, using the total probability theorem can be written as

$$
P\left(Z(i) \mid \mathrm{x}^{N}(i)\right)=\sum_{\forall E(i)} P\left(Z(i) \mid \mathrm{x}^{N}(i), E(i)\right) P\left(E(i) \mid \mathrm{x}^{N}(i)\right)
$$

where $\mathrm{x}^{N}(i)$ is the stack vector of $N$ targets given by

$$
\mathbf{x}^{N}(i)=\left[x^{1}(i), x^{2}(i), \ldots, x^{N}(i)\right]
$$


$E(i)$ is an association event (see Section 2.2). Then, term $P\left(Z(i) \mid \mathrm{x}^{N}(i), E(i)\right)$ is written as

$$
\begin{aligned}
P\left(Z(i) \mid \mathrm{x}^{N}(i), E(i)\right) & =P\left(Z(i) \mid \mathrm{x}^{N}(i), E(i), m_{i}\right) \\
& =\prod_{j=1}^{m_{i}} p\left(z_{j}(i) \mid x^{e_{j}(i)}(i)\right)
\end{aligned}
$$

where $P_{d}^{t}$ is the probability of detection of the $t$ th target. If the targets are identical then the detection probability may assumed to be equal.

The term $P\left(E(i) \mid \mathrm{x}^{N}(i)\right)$ can be also written as

$$
\begin{aligned}
P\left(E(i) \mid \mathrm{x}^{N}(i)\right) & =P\left(E(i) \mid \mathrm{x}^{N}(i), m_{i}\right) P\left(m_{i} \mid \mathrm{x}^{N}(i)\right) \\
& =P\left(E(i) \mid m_{i}\right) P\left(m_{i}\right)
\end{aligned}
$$

An association event $E(i)$ has $d(D(E(i)))$ number of target detections and $\left(m_{i}-\right.$ $d(D(E(i))))$ number of false alarms. Then, $P\left(E(i) \mid m_{i}\right)$ is given by

$$
\begin{gathered}
P\left(E(i) \mid m_{i}\right)=\frac{1}{P\left(m_{i}\right)} \frac{\left(m_{i}-d(D(E(i)))\right) !}{m_{i} !} \mu_{F A}\left(m_{i}-d(D(E(i)))\right) \\
\cdot \prod_{t=1}^{N}\left(P_{d}^{t}\right)^{D(E(i), t)}\left(1-P_{d}^{t}\right)^{(1-D(E(i), t))}
\end{gathered}
$$

Thus, the joint likelihood function of $N$ targets can be written as ( $\mathrm{R}$. Tharmarasa, 
T. Kirubarajan, M. L. Hernandez and A. Sinha, 2007)

$$
\begin{gathered}
P\left(Z(i) \mid \mathbf{x}^{N}(i)\right)=\sum_{\forall E(i)} \frac{\left(m_{i}-d(D(E(i)))\right) !}{m_{i} !} \mu_{F A}\left(m_{i}-d(D(E(i)))\right) \\
\cdot \prod_{t=1}^{N}\left(P_{d}^{t}\right)^{D(E(i), t)}\left(1-P_{d}^{t}\right)^{(1-D(E(i), t))} \prod_{j=1}^{m_{i}} p\left(z_{j}(i) \mid x^{e_{j}(i)}(i)\right)
\end{gathered}
$$

The $j$ th element of association event $E(i)$ is $e_{j}(i)$. If the value of $e_{j}(i)$ equals zero represents a false alarm, which is uniformly distributed in volume $V$ with pdf

$$
p\left(z_{j}(i) \mid x^{0}(i)\right)=\frac{1}{V}
$$

If the value of $e_{j}(i)$ equals $t$, means that the corresponding measurement is found with target $t$ pdf

$$
\begin{aligned}
& p\left(z_{j}(i) \mid x^{t}(i)\right)= \\
& (2 \pi)^{-\frac{k}{2}}|R(i)|^{-\frac{1}{2}} \exp \left(-\frac{1}{2}\left[z_{j}(i)-\mathbf{h}\left(x^{t}(i), y(i), i\right)\right] R(i)^{-1}\left[z_{j}(i)-\mathbf{h}\left(x^{t}(i), y(i), i\right)\right]^{\prime}\right)
\end{aligned}
$$

Then, the joint likelihood function can be rewritten as

$$
\begin{aligned}
& P\left(Z(i) \mid \mathbf{x}^{N}(i)\right)=\sum_{\forall E(i)} \frac{\left(m_{i}^{s}-d(D(E(i)))\right) !}{m_{i} !} \mu_{F A}\left(m_{i}-d(D(E(i)))\right) \\
& \cdot \prod_{t=1}^{N}\left(P_{d}^{t}\right)^{D(E(i), t)}\left(1-P_{d}^{t}\right)^{(1-D(E(i), t))}\left(\frac{1}{V}\right)^{d(D(E(i)))} \prod_{\substack{j=1 \\
e_{j}(i) \neq 0}}^{m_{i}} p\left(z_{j}(i) \mid x^{e_{j}(i)}(i)\right)
\end{aligned}
$$


The term

$$
\frac{\left(m_{i}-d(D(E(i)))\right) !}{m_{i} !} \mu_{F A}\left(m_{i}-d(D(E(i)))\right)\left(\frac{1}{V}\right)^{d(D(E(i)))}
$$

can reduced to

$$
\frac{\mu_{F A}\left(m_{i}\right)}{V^{m_{i}}} \cdot \frac{1}{\lambda^{d(D(E(i)))}}
$$

However,

$$
\frac{\mu_{F A}\left(m_{i}\right)}{V^{m_{i}}}
$$

is the likelihood function that all measurements at time $i$ are false alarms.

Thus, the joint likelihood ratio of a frame with $N$ targets is given by

$$
\begin{aligned}
& \Lambda\left(Z(i) \mid \mathrm{x}^{N}(i)\right)= \\
& \sum_{\forall E(i)} \frac{1}{\lambda^{d(D(E(i)))}} \prod_{t=1}^{N}\left(P_{d}^{t}\right)^{D(E(i), t)}\left(1-P_{d}^{t}\right)^{(1-D(E(i), t))} \prod_{\substack{j=1 \\
e_{j}(i) \neq 0}}^{m_{i}} p\left(z_{j}(i) \mid x^{e_{j}(i)}(i)\right)
\end{aligned}
$$

For a window, the joint log-likelihood ratio (JLLR) is given by

$$
\Phi\left[Z^{N_{w}}, \mathbf{x}^{N}\left(i_{0}\right)\right]=\sum_{i=i_{0}}^{i_{k}} \ln \left[\Lambda\left(Z(i) \mid \mathbf{x}^{N}(i)\right)\right]
$$


When amplitude information is incorporated then (3.7) can rewritten as

$$
\begin{aligned}
p\left(\left[z_{j}(i), a_{i}(j)\right] \mid x^{0}(i)\right) & =p\left(z_{j}^{s}(i) \mid x^{0}(i)\right) p_{0}^{\tau}\left(a_{j}(i)\right) \\
& =\frac{1}{V} \times \frac{1}{P_{f a}} a_{j}(i) \exp \left(-\frac{a_{j}(i)^{2}}{2}\right)
\end{aligned}
$$

Also, ( 3.8$)$ can be rewritten as

$$
p\left(\left[z_{j}(i), a_{i}(j)\right] \mid x^{t}(i)\right)=p\left(z_{j}(i) \mid x^{t}(i)\right) p_{1}^{\tau}\left(a_{j}(i)\right)
$$

Finally, the joint likelihood function in (3.12) becomes

$$
\begin{aligned}
& \Lambda\left([Z(i), a(i)] \mid \mathrm{x}^{N}(i)\right)= \\
& \quad \sum_{\forall E(i)} \frac{1}{\lambda^{d\left(D\left(E^{s}(i)\right)\right)}} \prod_{t=1}^{N}\left(P_{d}^{t}\right)^{D(E(i), t)}\left(1-P_{d}^{t}\right)^{(1-D(E(i), t))} \prod_{\substack{j=1 \\
e_{j}(i) \neq 0}}^{m_{i}} \rho_{j}(i) p\left(z_{j}(i) \mid x^{e_{j}(i)}(i)\right)
\end{aligned}
$$

Steps leading to ( 3.16$)$ are summarized below:

$$
\begin{aligned}
& P\left([Z(i), a(i)] \mid \mathbf{x}^{N}(i)\right)=\sum_{\forall E(i)} \frac{\left(m_{i}-d(D(E(i)))\right) !}{m_{i} !} \mu_{F A}\left(m_{i}-d(D(E(i)))\right) \\
& \cdot \prod_{\substack{t=1 \\
m_{i}}}^{N}\left(P_{D}^{t}\right)^{D(E(i), t)}\left(1-P_{D}^{t}\right)^{(1-D(E(i), t))}\left(\frac{1}{V}\right)^{d(D(E(i)))} \\
& \cdot \prod_{\substack{j=1 \\
e_{j}(i)=0}}^{m_{i}\left(a_{j}(i)\right)} \prod_{\substack{j=1 \\
e_{j}(i) \neq 0}}^{m_{i}} p_{1}^{\tau}\left(a_{j}(i)\right) p\left(z_{j}(i) \mid x^{e_{j}(i)}(i)\right)
\end{aligned}
$$




$$
\begin{aligned}
& P\left([Z(i), a(i)] \mid \mathbf{x}^{N}(i)\right)=\sum_{\forall E(i)} \frac{\left(m_{i}-d(D(E(i)))\right) !}{m_{i} !} \mu_{F A}\left(m_{i}-d(D(E(i)))\right) \\
& \cdot \prod_{t=1}^{N}\left(P_{d}^{t}\right)^{D(E(i), t)}\left(1-P_{D}^{t}\right)^{(1-D(E(i), t))} \\
& \quad \cdot\left(\frac{1}{V}\right)^{d(D(E(i)))} \prod_{j=1}^{m_{i}} p_{0}^{\tau}\left(a_{j}(i)\right) \prod_{\substack{j=1 \\
e_{j}(i) \neq 0}}^{m_{i}} \frac{p_{1}^{\tau}\left(a_{j}(i)\right)}{p_{0}^{\tau}\left(a_{j}(i)\right)} p\left(z_{j}(i) \mid x^{e_{j}(i)}(i)\right)
\end{aligned}
$$

The term

$$
\frac{\left(m_{i}-d(D(E(i)))\right) !}{m_{i} !} \mu_{F A}\left(m_{i}-d(D(E(i)))\right)\left(\frac{1}{V}\right)^{d(D(E(i)))} \prod_{j=1}^{m_{i}} p_{0}^{\tau}\left(a_{j}(i)\right)
$$

reduces to

$$
\frac{\mu_{f}\left(m_{i}\right)}{V^{m_{i}}} \frac{1}{\lambda^{d\left(D\left(E^{s}(i)\right)\right)}} \prod_{j=1}^{m_{i}} p_{0}^{\tau}\left(a_{j}(i)\right)
$$

The probability of all measurements being false alarms is given by

$$
\frac{\mu_{f}\left(m_{i}\right)}{V^{m_{i}}} \prod_{j=1}^{m_{i}} p_{0}^{\tau}\left(a_{j}(i)\right)
$$

\subsection{CJML-PDA Algorithms}

The JML-PDA is a multitarget parameter estimator, which is used here to initialize tracks of multiple targets, whose estimates and covariances are then fed to the JPDA tracker in a sliding window fashion. The novelty of this Combined JML-PDA and JPDA (CJML-PDA) tracker is in the sharing of information between the two tracking modules, which is different in previous batch-recursive approaches that treated the 
initialization and maintenance phases as two independent (and consecutive) stages of tracking. In real tracking problems, targets can enter and leave the surveillance region at any time. As a result, track initiation has to be considered at every sampling time. That is, track initialization does not occur during only the first few scan. Similarly, the fact that track maintenance stage has been activated does not obviate the need for further track initiations. Both have to be carried out simultaneously throughout the entire tracking interval. Because of this, the track initialization module (here, the JML-PDA) needs to take into account the number, states and qualities of the established tracks being retained by the track maintenance module (here, the JPDA). Otherwise, spurious tracks and track seduction will ensue, damaging the overall quality of the tracker.

The rest of the chapter organized as follows. Section 3.2 presents the formulation of the algorithm for any measurement and target spaces. Next, a restricted direct subspace search (RDSS) is presented to find estimates at global estimates using the CJML-PDA JLLR in Section 3.3. Sections 3.5 and 3.4 present, track validation and measurement gating for CJML-PDA. Finally, CRLB of CJML-PDA estimates are presented in Section 4.

The combined JML-PDA algorithm holds the same assumptions as in the JMLPDA. However, the following additional assumptions are made:

- An unknown number (assume $N^{*}$ ) of targets with $N$ number of them being confirmed in a batch with a given detection probability.

- All measurements are to be associated to new targets

The standard JPDA tracker assumes that the number of target is known $(N)$ and that of for each target a track has been formed (initiated). However, in the 
CJMKL-PDA tracker objective is to of track initialize tracks of any number $\left(N^{*}\right)$ of targets in a window. According to the above assumption, $N^{*}$ estimates need to be estimated using the CJML-PDA algorithm with the aid of JPDA. Thus, the required CJML-PDA JLLR based on $N^{*}$ number of targets can be written as

$$
\Phi\left[Z^{N_{w}} \mid\left(\mathrm{x}^{*}\right)^{N^{*}}\left(i_{0}\right)\right]=\left.\prod_{i=i_{0}}^{i_{k}}\left(\Phi\left[Z(i) \mid\left(\mathbf{x}^{*}\right)^{N^{*}}(i), \hat{\mathbf{x}}^{N}(i)\right]\right)\right|_{I\left(\hat{\mathrm{x}}^{N}(i)\right)}
$$

where $\mathrm{x}^{N}(i)$ and $\left(\mathrm{x}^{*}\right)^{N^{*}}\left(i_{0}\right)$ are stacked vectors of all confirmed and existing targets' states, respectively. In the above, $I\left(\hat{\mathrm{x}}^{N}(i)\right)$ is the information on $N$ targets in the JPDA tracker, whose incorporation in the JML-PDA, the main component in the combined algorithm.

Information from the JPDA are

- Number of existing targets $(N)$

- Validated measurements of the confirmed targets

- Track likelihoods

This information is then fed into the conventional JML-PDA to improve the detection capability.

In the JPDA the $t$ th target likelihood is

$$
p\left(z_{j}(i) \mid x^{t}(i)\right)=\mathcal{N}\left[z_{j}(i) ; \mathbf{h}\left(\hat{x}^{t}(i \mid i), y(i), i\right), S^{t}(i)\right]
$$

where $S^{t}(i)$ is the innovation covariance matrix given in (2.46). 
The expanded CJML-PDA JLLR ( 3.22) can be written as

$$
\begin{aligned}
\Phi\left[Z^{N_{w}} \mid\left(\mathrm{x}^{*}\right)^{N^{*}}\left(i_{0}\right)\right]= & \prod_{i=i_{0}}^{i_{k}}\left(\sum_{\forall E(i)} \frac{1}{\lambda^{d\left(D\left(E^{s}(i)\right)\right)}} \prod_{t=1}^{N}\left(P_{d}^{t}\right)^{D(E(i), t)}\left(1-P_{d}^{t}\right)^{(1-D(E(i), t))}\right. \\
& \left.\left.\prod_{\substack{j=1 \\
e_{j}(i) \neq 0}}^{m_{i}} p\left(z_{j}(i) \mid x^{e_{j}(i)}(i)\right)\right|_{p\left(z_{j}(i) \mid x \mathrm{old}_{(i))}\right.}\right)
\end{aligned}
$$

The single target CJML-PDA JLLR $\left(N^{*}=1\right)$, to extract the best estimate of a single target while there are $N$ already conformed tracks in the region can be found as

$$
\Phi\left[Z^{N_{w}} \mid x^{*}\left(i_{0}\right)\right]=\left.\prod_{i=i_{0}}^{i_{k}}\left(\Phi\left[Z(i) \mid \hat{\mathrm{x}}^{N}(i), x^{*}(i)\right]\right)\right|_{I\left(\hat{\mathrm{x}}^{N}(i)\right)}
$$

The new targets' estimate $\left(\hat{\mathrm{x}}^{*}\right)^{N^{*}}\left(i_{0}\right)$, is which maximizes the CJML-PDA JLLR function, can be found by

$$
\left(\hat{\mathrm{x}}^{N^{*}}\right)^{*}\left(i_{0}\right)=\underset{\left(\mathrm{x}^{*}\right)^{N *}\left(i_{0}\right)}{\arg \max } \Phi\left[Z^{N_{w}},\left(\mathrm{x}^{*}\right)^{N *}\left(i_{0}\right)\right]
$$

Tracks for the unknown number $N^{*}$ of targets are initialized at time $i_{0}$ if and only if they pass the track validation (see Section 3.4). A simple way to find $\hat{N}^{*}$ is to use the CJML-PDA repeatedly, starting with $N^{*}=1$ and then incrementing $N^{*}$ by 1 at until track validation is violated. 
The important steps in the CJML-PDA and JPDA tracker in a window are presented below:

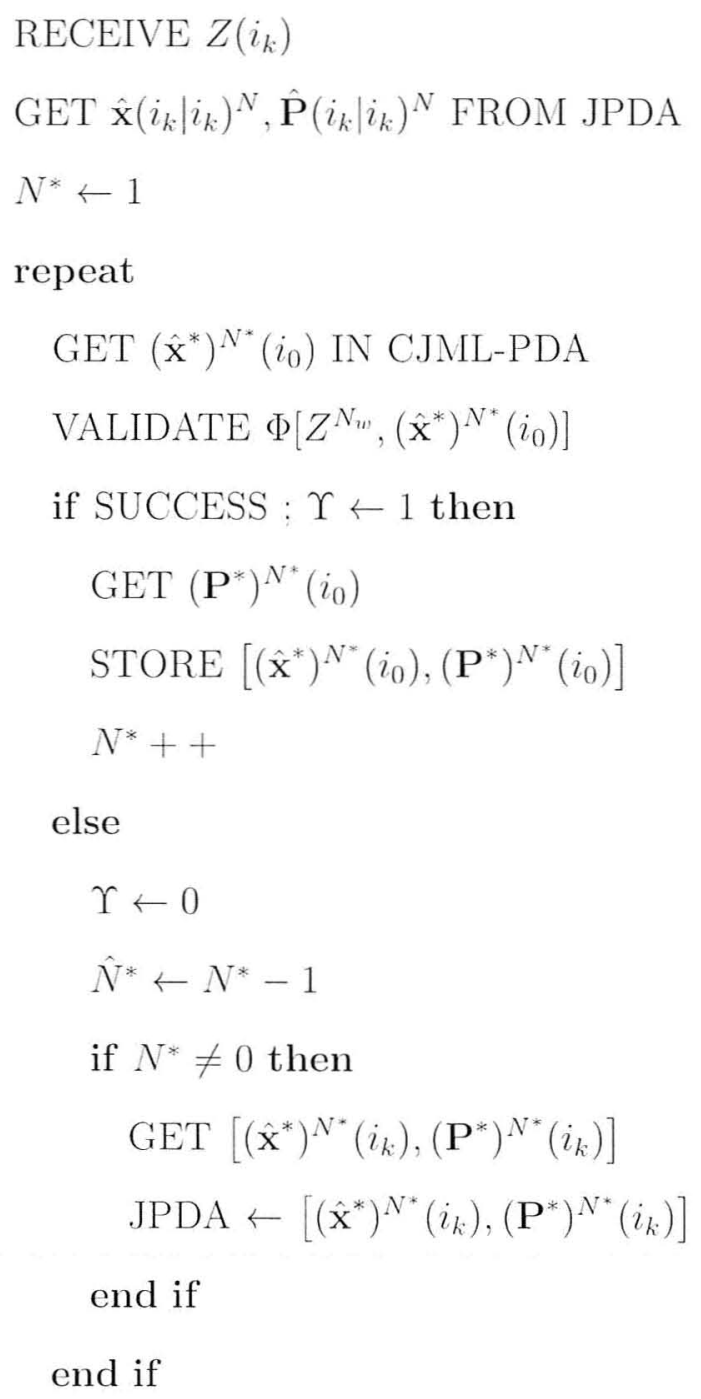

until $\Upsilon \neq 0$

DELETE $Z\left(i_{0}\right)$

$i_{k} \leftarrow i_{k}+1$ AND $i_{0} \leftarrow i_{0}+1$

New target extension is done with the CJML-PDA assumption a deterministic 
targets' motion model. The covariance $\mathbf{P}^{*}$ of the CJML-PDA estimate $\hat{\mathbf{x}}^{*}$ is detailed in Section 4.

Notice that the CJML-PDA approach 1 uses JPDA information up to the time $t_{k}$. Thus, CJML-PDA has to wait until JPDA completes it calculation in order to get the very recent ( $i_{k}$ th time) estimates. However, this can be avoided. One way is by using the JPDA predicted measures at the wanted time instead of real estimates. The other way is by adjusting the window length from $i_{0}$ to $i_{k}-1$. This ensures a simultaneous process on both CJML-PDA and JPDA.

\subsection{Log-Likelihood Ratio Optimization}

The track estimate in CJML-PDA is a parameter, which maximizes the CJML-PDA JLLR, is a highly non-convex function consisting of a large number of local maxima (possibly thousands for heavy clutter) and extended regions of near-zero gradient is difficult to optimize. Figure 3.3 shows the single target CJML-PDA JLLR surface for a batch of angle only measurements. The LLR plot with respect to target parameter position in $x$ and $y$ coordinates with a 4 -dimensional target parameter vector $(2$ position and 2 velocity components) at fixed velocity is considered.

Three techniques that have been used in CJML-PDA JLLR optimization: Genetic algorithm, multi-pass grid search and direct subspace search. In grid search, surveillance region is divided into multiple of regions to find the global LLR maximum via region wise local maximum. The number of grids is chosen based on measurement noise standard deviation. The prior works in (C. Jauffret and Y. Bar-Shalom, 1990), (T. Kirubarajan and Y. Bar-Shalom, 1996), (M. R. Chummun, Y. Bar-Shalom and T. Kirubarajan, 2002) have used multi-pass grid to get LLR global maximum. 
Genetic Search (GS) is a stochastic technique performed over a discrete parameter space using a rule based on biological evolution and survival of the fitness, (W. Blanding, P. Willett and Y. Bar-Shalom, 2007a). GS has seen little use in tracking community, and further a description about this method can be found in (Goldberg, 1989).

Directed Subspace Search (DSS) is an optimization tool to guide the search process in a way that identifies areas in parameter space that are more likely to contain local or global maxima. A real time tracker needs to obtain the global efficiently. Thus, in this report a restricted directed subspace search (RDSS) is introduced for fast process. In DSS, each measurement in $Z^{N_{w}}$ is converted to parameter space, and a maximization search is done according to those potential points. However in RDSS, measurements at reference time $\left(Z\left(i_{0}\right)\right)$ are converted to the parameter space to identify potential parameter points. RDSS type of mapping leaves more free parameters than of the DSS. Then, the free parameters are grided to find global maximum. Section 4.2 has this RDSS in $1 D$ parameter space and angle measurement space, which is also our test condition. Note that this method is not suitable for targets with very low probability of detection since the potential points are observed by only $Z\left(i_{0}\right)$.

\subsection{Track Validation}

C.JML-PDA always returns an estimate. Because of the nature of highly non-convex function, the optimization algorithm may converge into a local maximum resulting in a false track. Thus, a hypothesis testing is required to determine if there is a track and the result $\hat{\mathrm{x}}$ is the global maximum, to determine if it is due to a target or due to noise only measurement. 


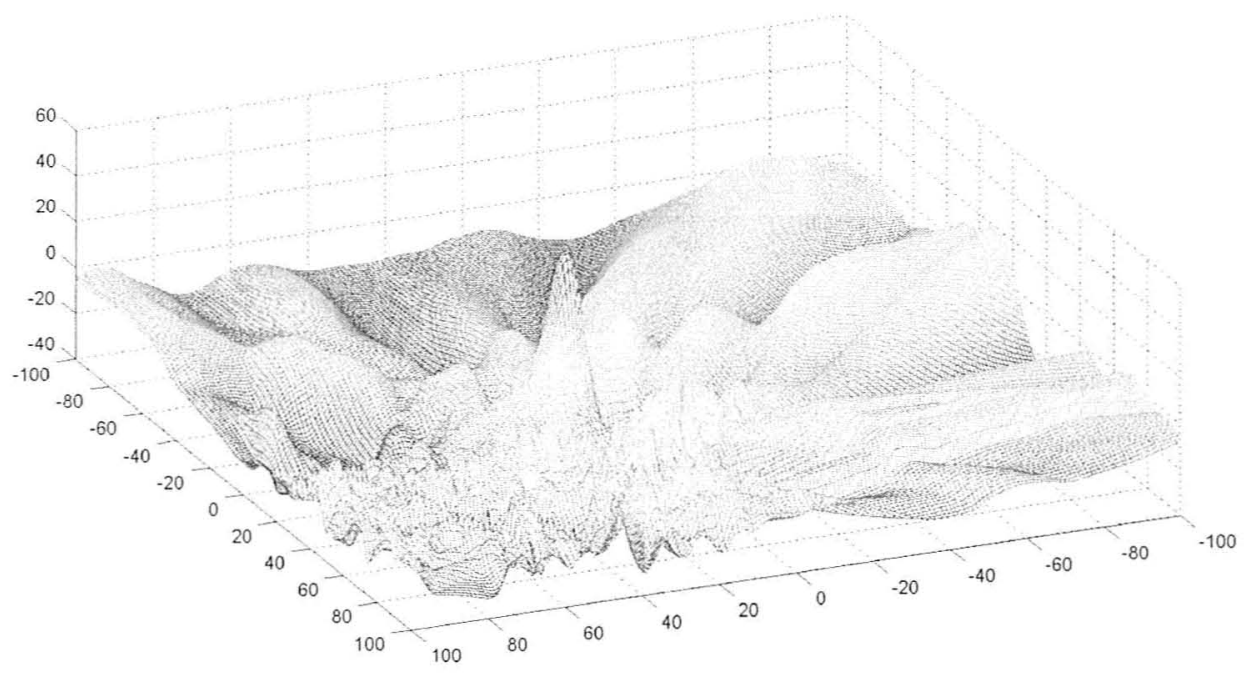

Figure 3.1: 2D view of 4D log-likelihood-ratio with known velocities and angle-only measurements. 
The estimate from the CJML-PDA JLLR is tested with hypothesis $H_{1}$ and $H_{0}$ hypothesis which are given by

$$
\begin{aligned}
& H_{1}=\{\text { here is a track and } \hat{\mathrm{x}} \text { is the global maximum }\} \\
& H_{0}=\{\text { There is no track }\}
\end{aligned}
$$

According to Neyman-Pearson lemma, the optimal test of hypothesis $H_{1}$ versus $H_{0}$ is by comparing CJML-PDA LLR at global maximum with a threshold $c_{\pi_{m}}$, and the threshold is selected by a predefined miss probability $\pi_{m}$

$$
\pi_{m}=\frac{1}{\sqrt{2 \pi}} \int_{-\infty}^{c_{\pi m}} e^{-\frac{\Lambda^{2}}{2}} d \Lambda
$$

where $\Lambda_{H_{1} / H_{0}}$ is the Gaussian test statistic defined in terms of $\mathbf{E}\left[\Phi\left[Z(i), H_{1}\right]\right]$ and $\mathbf{E}\left[\Phi\left[Z(i), H_{1}\right]^{2}\right]$. The first and second moment of log-likelihood ratio under hypothesis $H_{1}$, is given by

$$
\begin{aligned}
\Upsilon_{H_{1} / H_{0}} & =\sum_{i=1}^{i_{k}} \Upsilon_{H_{1} / H_{0}}(i) \\
& =\sum_{i=1}^{i_{k}}\left(\frac{\Phi[Z(i), \mathbf{x}]-\mathbf{E}\left[\Phi\left[Z(i), H_{1}\right]\right]}{\sqrt{\mathbf{E}\left[\Phi\left[Z(i), H_{1}\right]^{2}\right]}}\right)
\end{aligned}
$$

The $k^{\text {th }}$ moment of log-likelihood ratio under hypothesis $H_{1}$ is given by

$$
\begin{aligned}
& \mathbf{E}\left[\Phi\left[Z(i), H_{1}\right]^{k}\right]= \\
& \sum_{m_{i}=1}^{\infty} \int \ldots \int\left[\Phi\left[Z(i), H_{1}\right]\right]^{k} P(Z(i) \mid \mathbf{x}) \quad d Z(i)
\end{aligned}
$$


Finally, the track is accepted if

$$
\Upsilon_{H_{1} / H_{0}} \geq c_{\pi_{m}}
$$

\subsection{Measurement Gating}

Another way of reducing the computation time in CJML-PDA is by applying gating, which reduces the measurement set for consideration. Here, gating is set up on existing tracks based on Mahalanobis distance, which uses prior track estimate and its covariance. A measurement is called "validated measurement" if it satisfies

$$
\left(z_{j}(i)-\hat{z}^{t}(i \mid i-1)\right)^{\prime}\left(S(i)^{t}\right)^{-1}\left(z_{j}(i)-\hat{z}^{t}(i \mid i-1)\right) \leq \gamma
$$

$\hat{z}^{t}(i \mid i-1)$ is the predicted measurement of target $t$ given in 2.43. The term $S(i)^{t}$ is the innovation covariance of target $t$ at frame $i$, given in 2.46. $\gamma$ is the limiting threshold and chosen based on a desired probability of containing the target originated measurement within the gate.

Existing targets' measurements are validated before they are feed into CJML-PDA optimization and in the presented combined tracker this is done in the JPDA module. CJML-PDA assumes that the new targets could be anywhere in the survival region since there is no prior information about these targets. Thus, all measurements are associated in new targets.

To further understand, think of a two target scenario, that is $N=1$ and $N^{*}=1$. With possible gating on target 1 (assume it is the existing object) CJML-PDA LLR 
becomes (a similar equation can be found in (W. Blanding, P. Willett and Y. BarShalom, 2007b))

$$
\begin{aligned}
& \Lambda\left(Z^{N_{w}} \mid x^{*}\left(i_{0}\right)\right)=\sum_{i=i_{0}}^{i_{k}} \ln \left[\left(1-P_{d}^{1}\right)\left(1-P_{d}^{2}\right)\right. \\
& +\frac{P_{d}^{1}\left(1-P_{d}^{2}\right)}{\lambda} \sum_{j=1}^{b_{i}} p\left(\tilde{z}_{j}(i) \mid x^{1}(i)\right)+\frac{P_{d}^{2}\left(1-P_{d}^{1}\right)}{\lambda} \sum_{j=1}^{m_{i}} p\left(z_{j}(i) \mid x^{*}\left(i_{0}\right)\right) \\
& \left.+\frac{P_{d}^{1} P_{d}^{2}}{\lambda^{2}} \sum_{j=1}^{b_{i}} \sum_{\substack{l=1 \\
l \neq j}}^{m_{i}} p\left(\tilde{z}_{j}(i) \mid x^{1}(i)\right) p\left(z_{j}(i) \mid x^{*}\left(i_{0}\right)\right)\right]\left.\right|_{p\left(\tilde{z}_{j}(i) \mid x^{1}(i)\right) ; \forall i}
\end{aligned}
$$

where $\tilde{z}$ is the existing target's validated measurement, and there are $b_{i}$ number of such measurements in the set.

\subsection{CRLB and Covariance of the Estimates}

Multitarget Cramer-Rao Lower Bound (CRLB) calculation is more computationally complex than for single target. The single target case is computationally equivalent to well-separated multitarget case. Further, multitarget CRLB is worse in bearing-only case because, even if targets are well separated in state space they could be closely spaced in measurement space. In such an environment, the CRLB of the estimates is significantly different from that under single target condition., which is now reflects by the neighboring targets. Clearly, the inter-target distance has an impact on CRLB. Thus, the information reduction factor (IRF), which accounts for the loss of information due to association ambiguity, is not only in the function of probability of detection and probability of false alarm, but also a function of inter-target distance in measurement space. 
Let, $\mathrm{x}$ and $\hat{\mathrm{x}}$ be the standard true targets stack state vector, and their estimates respectively. Then, the error $\tilde{\mathrm{x}}$ in the estimate can be written as

$$
\tilde{\mathrm{x}}=\mathrm{x}-\hat{\mathrm{x}}
$$

The CRLB of an unbiased joint maximum likelihood estimate $\tilde{x}$ is given by

$$
\mathrm{E}\left[\tilde{\mathbf{x}} \tilde{\mathbf{x}}^{T}\right] \geq J^{-1}
$$

where $J$ is Fisher Information Matrix (FIM) is given by

$$
J=\left.\mathbf{E}\left\{\left[\nabla_{\mathbf{x}} \ln P\left(Z^{N_{w}} \mid \mathbf{x}\right)\right]\left[\nabla_{\mathbf{x}} \ln P\left(Z^{N_{w}} \mid \mathbf{x}\right)\right]^{\prime}\right\}\right|_{\mathbf{x}=\hat{\mathbf{x}}}
$$

For a valid track in CJML-PDA, the covariance of the estimated state is approximately equal to the CRLB bound. Thus, the covariance of the estimated state is given by

$$
\mathbf{P}\left(i_{0}\right)=\left(J^{N_{w}}\right)^{-1}
$$

where $J^{N_{w}}$ is the sum of Fisher information matrices given by

$$
J^{N_{w}}=\sum_{i=1}^{i_{k}} J(i)
$$


where

$$
J(i)=\left.E\left\{\left[\nabla_{\mathbf{x}} \ln P(Z(i) \mid \mathbf{x})\right]\left[\nabla_{\mathbf{x}} \ln P(Z(i) \mid \mathbf{x})\right]^{\prime}\right\}\right|_{\mathbf{x}=\hat{\mathbf{x}}}
$$

For the batch estimate CJML-PDA, the estimates in the window (CJML-PDA track) are driven by

$$
\hat{\mathbf{x}}(i)=\mathbf{f}\left(\hat{\mathbf{x}}\left(i_{0}\right), y(i), i-i_{0}\right)
$$

and the estimates' covariances are given by

$$
\mathbf{P}(i)=\mathbf{F}^{*}\left(\hat{\mathbf{x}}\left(i_{0}\right), y(i), i-i_{0}\right) \mathbf{P}\left(i_{0}\right) \mathbf{F}^{*}\left(\hat{\mathbf{x}}\left(i_{0}\right), y(i), i-i_{0}\right)
$$

Note that, for simulated data the FIM $J$ can be calculated from the true states. Therefore, for simulated scenario $J$ can be written as

$$
J=\left.E\left\{\left[\nabla_{\mathbf{x}} \ln P\left(Z^{N_{w}} \mid \mathrm{x}\right)\right]\left[\nabla_{\mathbf{x}} \ln P\left(Z^{N_{w}} \mid \mathbf{x}\right)\right]^{\prime}\right\}\right|_{\mathbf{x}=\mathbf{x}_{\text {true }}}
$$

\subsubsection{Information Reduction Factor : 1D Measurement Space}

The measurements in the validation region are taken into consideration in the calculation of the Information Reduction Factor (IRF).

With $m_{i}$ angle measurements, $J_{i}$ can be written as an $m$-fold integral form,

$$
J_{i}=\sum_{m_{i}=1}^{\infty} \int \ldots \int\left[\frac{\nabla_{x} P(Z(i) \mid x)}{P(Z(i) \mid x)}\right]\left[\frac{\nabla_{x} P(Z(i) \mid x)}{P(Z(i) \mid x)}\right]^{\prime} P(Z(i) \mid x) \quad d Z(i)
$$


In our simulation, track initialization using CJML-PDA has two phases. Single target initialization ( 4.5 ) and single target initialization in the presence of previous targets (4.7).

The calculation of the CRLB includes the IRF that reflects the loss of information due to false alarms, missed detections and the presence of multiple targets. In the multitarget CRLB, the proximity of negibouring targets is factored as a normalized inner-target distance in measurement space in the calculation of IRF.

Consider the case two where $x^{*}$ and $x$ are new and existing target dynamic states respectively. Thus, the FIM at time $i$ can be written as

$$
J_{i}=\sum_{m_{i}=1}^{\infty} \int \ldots \int\left[\frac{\left.\nabla_{x^{*}} P\left(Z(i) \mid x^{*}\right)\right]}{P\left(Z(i) \mid x^{*}\right)}\right]\left[\frac{\nabla_{x^{*}} P\left(Z(i) \mid x^{*}\right)}{P\left(Z(i) \mid x^{*}\right)}\right]^{\prime} P\left(Z(i) \mid x^{*}\right) \quad d Z(i)
$$

The term $\nabla_{x^{*}} P\left(Z(i) \mid x^{*}\right)$ can be found using

$$
\begin{gathered}
\nabla_{x^{*}} P\left(Z(i) \mid x^{*}\right)=\frac{\mu_{f}\left(m_{i}\right)}{V^{m_{i}}}\left\{\frac{P_{d}^{*}\left(1-P_{d}\right)}{\lambda \sqrt{2 \pi} \sigma_{\theta}}\right. \\
\cdot \sum_{j=1}^{m_{i}} \exp \left(-\frac{\left(z_{j}(i)-\mathbf{h}\left(x^{*}(i), y(i), i\right)\right)}{2 \sigma_{\theta}^{2}}\right) \frac{z_{j}(i)-\mathbf{h}\left(x^{*}(i), y(i), i\right)}{\sigma_{\theta}^{2}}\left[\nabla_{x^{*}} \mathbf{h}\left(x^{*}(i), y(i), i\right)\right] \\
+\frac{P_{d}^{*} P_{d}}{\lambda^{2} 2 \pi \sigma_{0}^{2}} \sum_{j=1}^{m_{i}} \sum_{\substack{l=1 \\
l \neq j}}^{m_{i}} \exp \left(-\frac{\left(z_{j}(i)-\mathbf{h}\left(x^{*}(i), y(i), i\right)\right)}{2 \sigma_{0}^{2}}-\frac{\left(z_{l}(i)-\mathbf{h}(x(i), y(i), i)\right)}{2 \sigma_{0}^{2}}\right) \\
\left.. \frac{z_{j}(i)-\mathbf{h}\left(x^{*}(i), y(i), i\right)}{\sigma_{\theta}^{2}}\left[\nabla_{x^{*}} \mathbf{h}\left(x^{*}(i), y(i), i\right)\right]\right\}
\end{gathered}
$$


Now, introduce a new variable $\xi_{i}$ such that

$$
\xi_{i}=\left[\begin{array}{l}
\xi_{i 1} \\
\cdot \\
\xi_{i j} \\
\cdot \\
\cdot \\
\xi_{i m_{i}}
\end{array}\right]=\left[\begin{array}{l}
\frac{\left(z_{1}(i)-\mathbf{h}\left(x^{*}(i), y(i), i\right)\right)}{\sigma_{\theta}} \\
\cdot \\
\frac{\left(z_{j}(i)-\mathbf{h}\left(x^{*}(i), y(i), i\right)\right)}{\sigma_{\theta}} \\
\cdot \\
\frac{\left(z_{m_{i}}(i)-\mathbf{h}\left(x^{*}(i), y(i), i\right)\right)}{\sigma_{\theta}}
\end{array}\right]
$$

The term corresponding to the second target, $\frac{\left(z_{j}(i)-\mathbf{h}(x(i), y(i), i)\right)}{\sigma_{0}}$, is given by

$$
\begin{aligned}
\frac{\left(z_{j}(i)-\mathbf{h}(x(i), y(i), i)\right)}{\sigma_{\theta}} & =\frac{\left(z_{j}(i)-\mathbf{h}\left(x^{*}(i), y(i), i\right)+\mathbf{h}\left(x^{*}(i), y(i), i\right)-\mathbf{h}(x(i), y(i), i)\right)}{\sigma_{\theta}} \\
& =\xi_{i j}+d\left(\mathbf{h}\left(x^{*}(i), y(i), i\right), \mathbf{h}\left(x^{*}(i), y(i), i\right), \sigma_{\theta}\right) \\
& =\xi_{i j}+d_{i}(1,2)
\end{aligned}
$$

where $d\left(\mathbf{h}\left(x^{*}(i), y(i), i\right), \mathbf{h}\left(x^{*}(i), y(i), i\right), \sigma_{\theta}\right)$ is a normalized distance between the targets (normalized inner-target distance) in the measurement space. To simplify the notation, it is denoted as $d_{i}(1,2)$.

The differential term $\frac{d \xi_{i j}}{d z_{j}(i)}$ in integral is given by

$$
d \xi_{i j}=\frac{d z_{j}(i)}{\sigma_{\theta}}
$$


With the variable transformation, gradient can be rewritten as

$$
\begin{gathered}
\nabla_{x^{*}} P\left(Z(i) \mid x^{*}\right)=\frac{\mu_{f}\left(m_{i}\right)}{V^{m_{i}}}\left\{\frac{P_{d}^{*}\left(1-P_{d}\right)}{\lambda \sqrt{2 \pi} \sigma_{\theta}} \sum_{j=1}^{m_{i}} \exp \left(-\frac{\xi_{i j}^{2}}{2}\right) \xi_{i j} \frac{1}{\sigma_{\theta}}\left[\nabla_{x^{*}} \mathbf{h}\left(x^{*}(i), y(i), i\right)\right]\right. \\
\left.+\frac{P_{d}^{*} P_{d}}{\lambda^{2} 2 \pi \sigma_{\theta}^{2}} \sum_{j=1}^{m_{i}} \sum_{\substack{l=1 \\
l \neq j}}^{m_{i}} \exp \left(-\frac{\xi_{i j}^{2}}{2}-\frac{\left(\xi_{i l}+d_{\theta_{i}}\right)^{2}}{2}\right) \xi_{i j} \frac{1}{\sigma_{\theta}}\left[\nabla_{x^{*}} \mathbf{h}\left(x^{*}(i), y(i), i\right)\right]\right\}
\end{gathered}
$$

Then $j_{i}$ can be written as

$$
J_{i}=\sum_{m_{i}=1}^{\infty} \sigma_{\theta}^{m_{i}} \int \ldots \int\left[\frac{\left.\nabla_{x^{*}} P\left(\xi_{i} \mid x^{*}\right)\right]}{P\left(\xi_{i} \mid x^{*}\right)}\right]\left[\frac{\nabla_{x^{*}} P\left(\xi_{i} \mid x^{*}\right)}{P\left(\xi_{i} \mid x^{*}\right)}\right]^{\prime} P\left(\xi_{i} \mid x^{*}\right) \quad d \xi_{i}
$$

For one dimensional measurement space $z_{j}(i)$ is a scalar. Therefore, the above integral of $J_{i}$ can be rewritten as

$$
\begin{aligned}
& J_{i}=\sum_{m_{i}=1}^{\infty} \frac{\mu_{f}\left(m_{i}\right)}{V^{m_{i}}} \sigma_{\theta}^{m_{i}} \int_{\tau}^{\infty} \cdots \int_{\tau}^{\infty} \int_{-g}^{+g} \cdots \int_{-g}^{+g} \\
& \times \frac{\left(\sum_{j=1}^{m_{i}} \exp \left(-\frac{\xi_{i j}^{2}}{2}\right) \xi_{i j}\left(\frac{P_{d}^{*}\left(1-P_{d}\right)}{\lambda \sqrt{2 \pi} \sigma_{\theta}}+\frac{P_{d}^{*} P_{d}}{\lambda^{2} 2 \pi \sigma_{\theta}^{2}} \sum_{\substack{l=1 \\
l \neq j}}^{m_{i}} \exp \left(-\frac{\left(\xi_{i l}+d_{i}(1,2)\right)^{2}}{2}\right)\right)\right)^{2}}{\left(1-P_{d}^{*}\right)\left(1-P_{d}\right)+\frac{1}{\lambda \sqrt{2 \pi} \sigma_{0}} \sum_{j=1}^{m_{i}}\left(P_{d}\left(1-P_{d}^{*}\right) \exp \left(-\frac{\left(\xi_{i j}+d_{i}(1,2)\right)^{2}}{2}\right) \cdots\right.} \cdot \cdot \\
& \cdots \frac{\cdots}{\left.\cdots+P_{d}^{*}\left(1-P_{d}\right) \exp \left(-\frac{\xi_{i j}^{2}}{2}\right)\right)+\frac{P_{D}^{*} P_{D}}{\lambda^{2} 2 \pi \sigma_{0}^{2}} \sum_{j=1}^{m_{i}} \sum_{\substack{l=1 \\
l \neq j}}^{m_{i}} \exp \left(-\frac{\xi_{i j}^{2}}{2}-\frac{\left(\xi_{i l}+d_{i}(1,2)\right)^{2}}{2}\right)} d a_{i} d \xi_{i} \\
& \times \frac{1}{\sigma_{\theta}^{2}}\left[\nabla_{x^{*}} \mathbf{h}\left(x^{*}(i), y(i), i\right)\right]\left[\nabla_{x^{*}} \mathbf{h}\left(x^{*}(i), y(i), i\right)\right]^{\prime}
\end{aligned}
$$


The IRF be denoted by $\mathcal{Q}$ is given by

$$
\begin{aligned}
& \mathcal{Q}_{i}\left(P_{d}, P_{f a}, \sigma_{\theta}, d_{i}(1,2)\right)=\sum_{m_{i}=1}^{\infty} \frac{\mu_{f}\left(m_{i}\right)}{V^{m_{i}}} \sigma_{\theta}^{m_{i}} \int_{\tau}^{\infty} \ldots \int_{\tau}^{\infty} \int_{-g}^{+g} \ldots \int_{-g}^{+g} \\
& \times \frac{\left(\sum_{j=1}^{m_{i}} \exp \left(-\frac{\xi_{i j}^{2}}{2}\right) \xi_{i j}\left(\frac{P_{d}^{*}\left(1-P_{d}\right)}{\lambda \sqrt{2 \pi} \sigma_{\theta}}+\frac{P_{d}^{*} P_{d}}{\lambda^{2} 2 \pi \sigma_{\theta}^{2}} \sum_{\substack{l=1 \\
l \neq j}}^{m_{i}} \exp \left(-\frac{\left(\xi_{i l}+d_{i}(1,2)\right)^{2}}{2}\right)\right)\right)^{2}}{\left(1-P_{d}^{*}\right)\left(1-P_{d}\right)+\frac{1}{\lambda \sqrt{2 \pi} \sigma_{\theta}} \sum_{j=1}^{m_{i}}\left(P_{d}\left(1-P_{d}^{*}\right) \exp \left(-\frac{\left(\xi_{i j}+d_{i}(1,2)\right)^{2}}{2}\right) \cdots\right.} \cdot \cdots \\
& \quad \cdots \quad d a_{i} d \xi_{i} \\
& \left.\quad \cdots+P_{d}^{*}\left(1-P_{d}\right) \exp \left(-\frac{\xi_{i j}^{2}}{2}\right)\right)+\frac{P_{D}^{*} P_{D}}{\lambda^{2} 2 \pi \sigma_{\theta}^{2}} \sum_{j=1}^{m_{i}} \sum_{\substack{l=1 \\
l \neq j}}^{m_{i}} \exp \left(-\frac{\xi_{i j}^{2}}{2}-\frac{\left(\xi_{i l}+d_{i}(1,2)\right)^{2}}{2}\right)
\end{aligned}
$$

Thus, $J_{i}$ can be written as

$$
J_{i}=\mathcal{Q}_{i}\left(P_{d}, P_{f a}, \sigma_{\theta}, d_{i}(1,2)\right) \times \frac{1}{\sigma_{\theta}^{2}}\left[\nabla_{x^{*}} \mathbf{h}\left(x^{*}(i), y(i), i\right)\right]\left[\nabla_{x^{*}} \mathbf{h}\left(x^{*}(i), y(i), i\right)\right]^{\prime}
$$

For the test scenario, $\nabla_{x^{*}} \mathbf{h}\left(x^{*}(i), y(i), i\right)$ is given by

$$
\nabla_{x^{*}} \mathbf{h}\left(x^{*}(i), y(i), i\right)=\left[\begin{array}{c}
\frac{\partial \theta_{i}\left(x^{*}\right)}{\partial \xi} \\
\frac{\partial \theta_{i}\left(x^{*}\right)}{\partial \dot{\xi}}
\end{array}\right]
$$

For any single scan estimator, the above term can be found by

$$
\nabla_{x} \theta_{i}\left(x^{*}\right)=\frac{1}{1+\left(\frac{h^{s}(i)}{\xi(i)-\xi^{s}(i)}\right)^{2}} \cdot \frac{-h^{s}(i)}{\left(\xi(i)-\xi^{s}(i)\right)^{2}}\left[\begin{array}{l}
1 \\
0
\end{array}\right]
$$


For the batch estimator CJML-PDA the corresponding term is given by,

$$
\nabla_{x} \theta_{i}\left(x^{*}\right)=\frac{1}{1+\left(\frac{h^{s}(i)}{\xi\left(i_{0}\right)+\left(i-i_{0}\right) \dot{\xi}\left(i_{0}\right)-\xi^{s}(i)}\right)^{2}} \cdot \frac{-h^{s}(i)}{\left(\xi\left(i_{0}\right)+\left(i-i_{0}\right) \dot{\xi}\left(i_{0}\right)-\xi^{s}(i)\right)^{2}}\left[\begin{array}{c}
1 \\
i-i_{0}
\end{array}\right]
$$

As seen in (3.55), the information reduction factor $(\mathcal{Q})$ is not only function of $P_{d}, P_{F a}$ and $\sigma_{\theta}$ but also a function of $d_{i}(i, 2)$, the normalized inter-target distance in measurement space. The IRF values for different $P_{d}, P_{f a}$ and $d_{i}(i, 2)$ are summarized in Table 3.1 for a fixed sensor standard deviation.

\begin{tabular}{|c|c|c|c|c|c|}
\hline$P_{d}$ & $P_{f a}$ & $d_{i}=0$ & $d_{i}=3$ & $d_{i}=6$ & $d_{i}=9$ \\
\hline 0.8 & 0.1 & 0.3594 & 0.6797 & 0.7173 & 0.7243 \\
\hline 0.9 & 0.1 & 0.4051 & 0.7312 & 0.7500 & 0.7866 \\
\hline 0.9 & 0.15 & 0.3879 & 0.7011 & 0.7501 & 0.7675 \\
\hline
\end{tabular}

The above approach can be extended to find the $k^{\text {th }}$ moment of log-likelihood ratio under test hypotheses $H_{1}$. The equation 3.30 values as function of normalized distance are summarized in Table 3.2 .

Table 3.2: Mean and standard deviation (s.d.) under test hypotheses $H_{1}$ for fixed $\sigma_{0}=0.02(\mathrm{rad})$

\begin{tabular}{ccccccc}
\hline \hline$P_{d}$ & $P_{f a}$ & Moments & $d_{i}=0$ & $d_{i}=3$ & $d_{i}=6$ & $d_{i}=9$ \\
\hline \multirow{2}{*}{0.8} & \multirow{2}{*}{0.1} & mean & 9.9779 & 9.3133 & 9.2758 & 9.2206 \\
& & s.d. & 11.3281 & 10.6821 & 10.6555 & 10.6128 \\
\hline \multirow{2}{*}{0.9} & \multirow{2}{*}{0.1} & mean & 11.4763 & 10.8243 & 10.7675 & 10.5585 \\
& & s.d. & 12.5989 & 11.9540 & 11.9142 & 11.7858 \\
\hline \multirow{2}{*}{0.9} & \multirow{2}{*}{0.15} & mean & 10.3140 & 9.7930 & 9.7062 & 9.6675 \\
& & s.d. & 11.6083 & 11.0468 & 10.9841 & 10.9598 \\
\hline \hline
\end{tabular}


The single target IRF is same as the case where targets are well separated. Thus, IRF single target can be found using

$$
\mathcal{Q}_{\text {single }}=\mathcal{Q}_{d_{i}(1,2) \rightarrow \infty}
$$




\section{Chapter 4}

\section{Simulation Studies}

\subsection{Test Scenario}

Angle-only tracking is a challenging estimation problem because of the nonlinearity in the measurement model. Therefore, to ensure the target's observability the sensor's platform needs relative maneuvers or acceleration. Also, due to low information content of the measurement there can be high estimation error. Higher false alarm rate makes the condition even worse.

The simulation study was conducted on an extended version of the scenario in $\mathrm{Y}$. Bar-Shalom, X. R. Li and T. Kirubarajan, 2001, pg. 387 to tracking two neighbouring targets with an angle-only sensor. Both the sensor and targets follow a constant velocity motion model, but the sensor at a constant altitude while the targets are on the ground. This ensures observability.

A target dynamic state donated with position and velocity at time $i$ is given by

$$
x^{t}(i)=\left[\begin{array}{ll}
\xi^{t}(i) & \dot{\xi}^{t}(i)
\end{array}\right]
$$


where $\xi^{t}(i)$ and $\dot{\xi}_{n}(i)$ are the position and velocity, respectively.

Target motion matrix $\mathbf{F}$ for the above target state can be expressed as

$$
x^{t}(i+1)=\left[\begin{array}{ll}
1 & T \\
0 & 1
\end{array}\right] x^{t}(i)+\left[\begin{array}{c}
\frac{T^{2}}{2} \\
T
\end{array}\right] \sigma_{t}^{2}(i)
$$

It is assumed that $E\left[\nu_{n}(i) \nu_{n}(i)^{\prime}\right]=\sigma_{t}^{2}$.

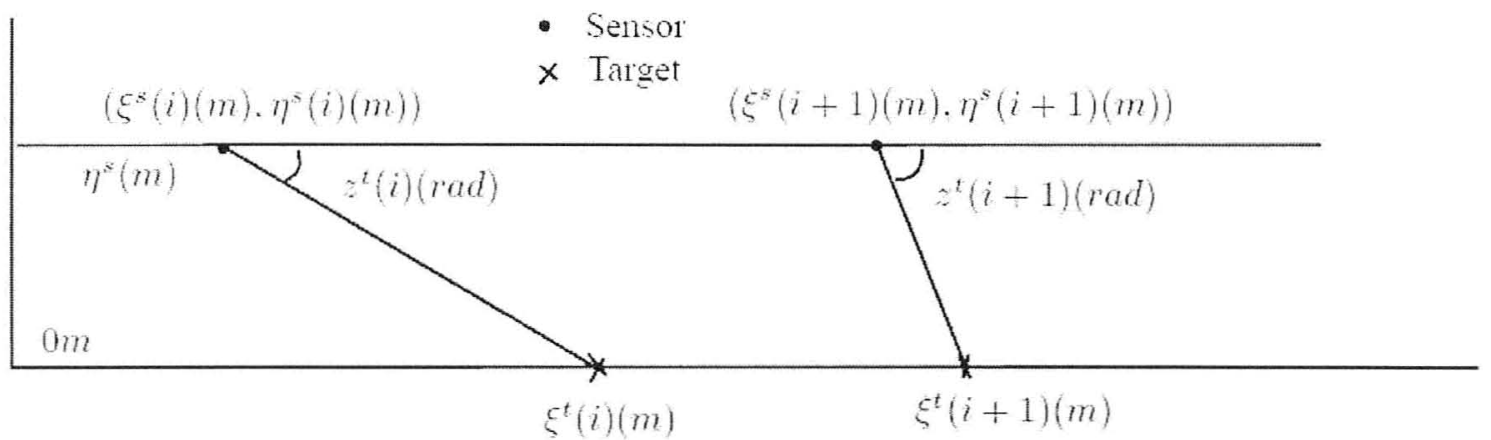

Figure 4.1: Target and sensor motion ( Y. Bar-Shalom, X. R. Li and T. Kirubarajan, 2001, pg. 388)

The sensor platform is assumed to be moving horizontal with constant velocity. Figure 4.1 illustrates target and sensor motion for time $i$ to $i+1$ (discrete). The state of the sensor platform can be defined by

$$
y^{s}(i)=\left[\begin{array}{lll}
\xi^{s}(i) & \dot{\xi}^{s}(i) & \eta^{s}(i)
\end{array}\right]
$$

where $\eta^{s}(i)$ is the altitude of the platform. 
Angle-only measurements are taken by the sensor. Thus, a target generated measurement can written as

$$
z^{t}(i)=\tan ^{-1}\left(\frac{\eta^{s}(i)}{\xi^{t}(i)-\xi^{s}(i)}\right)+\varepsilon_{i}
$$

Simulation studies were done on two neighbouring targets. Thus, there are three possible circumstances in track initialization:

- Single target initialization

- Two targets initialization (jointly estimate two targets states)

- Single target initialization in the presence of an existing target

The LLR with respect to single target state can be written as

$$
\Phi\left[Z^{N_{w}}, x^{1}\left(i_{0}\right)\right]=\sum_{i=i_{0}}^{i_{k}} \ln \left[\left(1-P_{d}^{1}\right)+\frac{P_{d}^{1}}{\lambda} \sum_{j=1}^{m_{i}} p\left(z_{j}(i) \mid x^{1}(i)\right)\right]
$$

The JLLR of two new targets can written as

$$
\begin{gathered}
\Phi\left[Z^{N_{w}}, x^{1}\left(i_{0}\right), x^{2}\left(i_{0}\right)\right]=\sum_{i=i_{0}}^{i_{k}} \ln \left[\left(1-P_{d}^{1}\right)\left(1-P_{d}^{2}\right)+\frac{P_{d}^{1}\left(1-P_{d}^{2}\right)}{\lambda} \sum_{j=1}^{m_{i}} p\left(z_{j}(i) \mid x^{1}(i)\right)\right. \\
\left.+\frac{P_{d}^{2}\left(1-P_{d}^{1}\right)}{\lambda} \sum_{j=1}^{m_{i}} p\left(z_{j}(i) \mid x^{2}(i)\right)+\frac{P_{d}^{1} P_{d}^{2}}{\lambda^{2}} \sum_{j=1}^{m_{i}} \sum_{\substack{l=1 \\
l \neq j}}^{m_{i}} p\left(z_{j}(i) \mid x^{1}(i)\right) p\left(z_{j}(i) \mid x^{2}(i)\right)\right] \quad \text { (4.6) }
\end{gathered}
$$

In the third case, single target initialization presence of a target, JLLR can be 
written as

$$
\begin{gathered}
\Phi\left[Z^{N_{w}}, x^{2}\left(i_{0}\right)\right]=\sum_{i=i_{0}}^{i_{k}} \ln \left[\left(1-P_{d}^{1}\right)\left(1-P_{d}^{2}\right)+\frac{P_{d}^{1}\left(1-P_{d}^{2}\right)}{\lambda} \sum_{j=1}^{b_{i}} p\left(z_{j}(i) \mid x^{1}(i)\right)\right. \\
\left.+\frac{P_{d}^{2}\left(1-P_{d}^{1}\right)}{\lambda} \sum_{j=1}^{m_{i}} p\left(z_{j}(i) \mid x^{2}(i)\right)+\frac{P_{d}^{1} P_{d}^{2}}{\lambda^{2}} \sum_{j=1}^{b_{i}} \sum_{\substack{l=1 \\
l \neq j}}^{m_{i}} p\left(z_{j}(i) \mid x^{1}(i)\right) p\left(z_{j}(i) \mid x^{2}(i)\right)\right]\left.\right|_{p\left(z_{j}(i) \mid x^{1}(i)\right)}
\end{gathered}
$$

where $x^{*}\left(i_{0}\right)$ and $x\left(i_{0}\right)$ are new target and existing target states, respectively.

\subsection{Restricted Direct Subspace Search (RDSS)}

Position grids are calculated from measurements to reduce computational load. Instead of searching the whole surveillance region, the likelihood calculation is done only at those potential points. Velocity component that cannot be generated from measurement set $Z\left(i_{0}\right)$ are considered as free component. Therefore, velocity is bounded between a possible minimum and maximum.

For the above scenario, the $z_{j}$ th measurement's position estimate can be written as

$$
\hat{\xi}\left(z_{j}\right)=\frac{\eta^{s}}{\tanh z_{j}}+\xi^{s}
$$

The the effectiveness of DSS algorithm could be improved by not restricting components to the mapped parameters, but also provide a range as in (W. R. Blanding, P. K. Willett, Y. Bar-Shalom and R. Lynch, 2008). Therefore, the RDSS estimates are bounded according to the estimate's variance. This is to avoid the errors at those 
potential points due to the sensor noise. The variance of estimate $\xi\left(z_{j}\right)$ is given by, ( Y. Bar-Shalom, X. R. Li and T. Kirubarajan, 2001, pg. 140). Then

$$
E\left[\tilde{\xi}\left(z_{j}\right)^{2}\right]=\frac{1}{\sigma_{\theta}}\left(\frac{\partial \mathbf{h}}{\partial \xi}\right)^{2}
$$

where

$$
\frac{\partial \mathbf{h}}{\partial \xi}=\frac{\frac{1}{\sigma_{\theta}}}{1+\left(\frac{\eta^{s}}{\xi\left(z_{j}\right)-\xi^{s}}\right)^{2}} \cdot \frac{-\eta^{s}}{\left(\xi\left(z_{j}\right)-\xi^{s}\right)^{2}}
$$

The $95 \%$ confidence region of the estimate is considered as a grid to optimize CJML-PDA. 


\section{Chapter 5}

\section{Numerical Results}

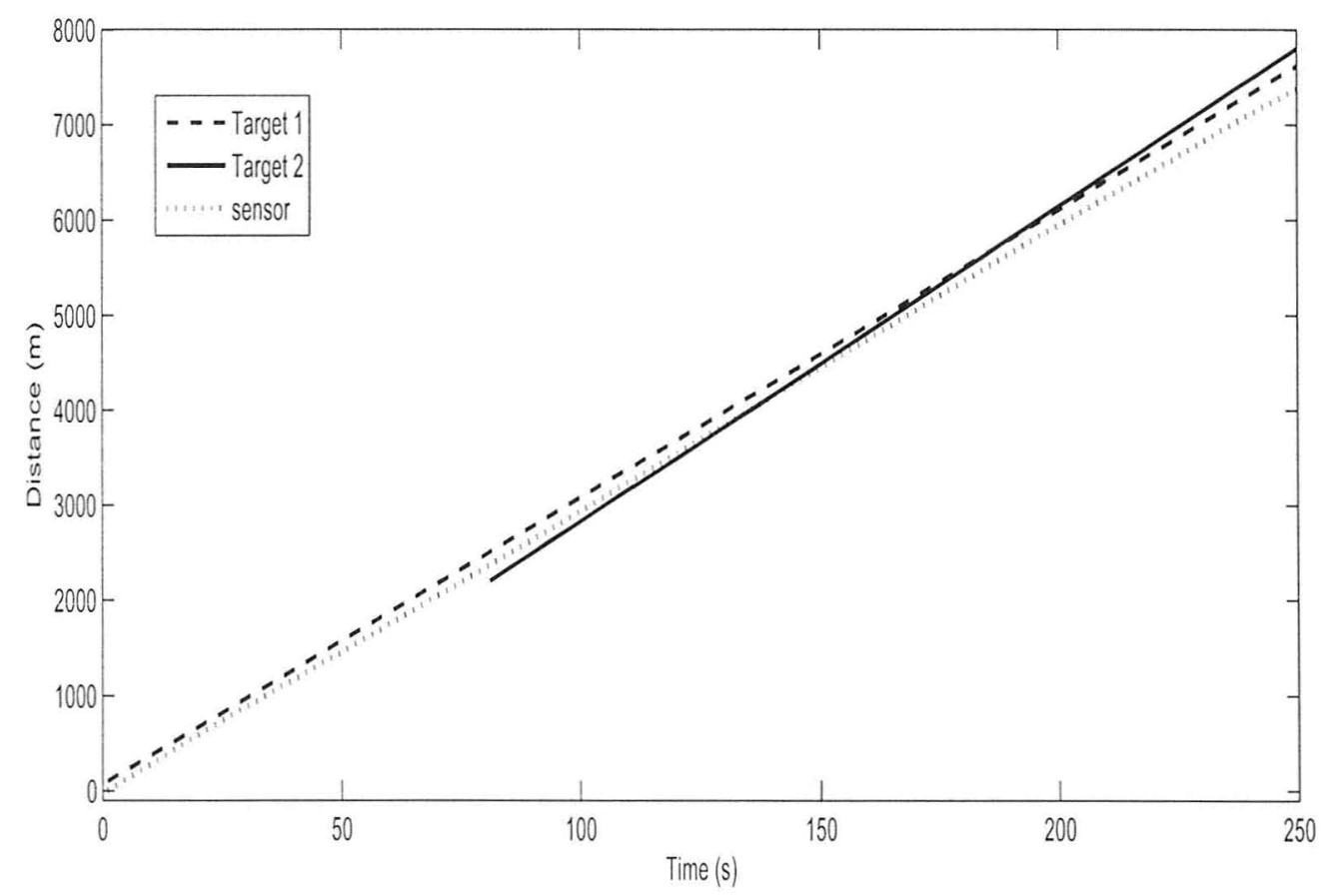

Figure 5.1: Target and sensor trajectories.

Simulated target trajectories are shown in Figure 5.1, where the targets cross 
Table 5.1: Scenario Parameters

\begin{tabular}{|l|l|}
\hline \multicolumn{2}{|c|}{ Parameter File } \\
\hline$P_{d}^{1}=P_{d}^{2}$ & 0.9 \\
$P_{f a}$ & 0.1 \\
$\sigma_{\theta}$ (sensor noise s.d.) & $0.02 \mathrm{rad}$ \\
avg. no. of false alarms & 12 \\
$T$ (sampling time) & $4 \mathrm{~s}$ \\
$\sigma_{t}$ (process noise s.d.) & $0.05 \mathrm{~m} / \mathrm{s}$ \\
target 1 starting time of & $1 \mathrm{~s}$ \\
$x^{1}(1)$ (initial state) & {$[100 \mathrm{~m} \quad 30 \mathrm{~m} / \mathrm{s}]$} \\
target 2 starting time of & $81 \mathrm{~s}$ \\
$x^{2}(81)$ (initial state) & {$[2200 \mathrm{~m} \quad 33 \mathrm{~m} / \mathrm{s}]$} \\
$y^{1}$ (1) (initial state) & {$[0 \mathrm{~m} \quad 31 \mathrm{~m} / \mathrm{s} \quad 500 \mathrm{~m}]$} \\
$V$ & {$[0, \pi]$} \\
Total time & $300 \mathrm{~s}$ \\
No. of Monte-Carlo runs & 100 \\
$N_{w}$ (Number of frames) & 10 \\
$\pi_{m}$ (miss probability) & $5 \%$ \\
$c_{\pi_{m}}$ (confidence region) & $95 \%$ \\
\hline
\end{tabular}

one another and they are closely-spaced. Initially the process noise is a very low value. This is increased after some time. The platform is kept at a fixed altitude and assumed to be moving parallel to targets.

Table 5.1 provides the scenario parameters. On average 12 false alarms are presented in each scan. Figure 5.2 shows the estimated trajectories of Target 2 over 100 Monte Carlo runs in both CJML-PDA and JPDA modules, respectively. It can be seen that tracks are within the $95 \%$ confidence region, meaning that the proposed estimator mects the CRLB. That is thae estimator is efficient. The same nature was also found for the velocity estimates as shown in Figure 5.3.

Figures 5.4 and 5.5 show the Root Mean Squared Errors (RMSE) in position and velocity estimates of both targets, respectively. Also, the figures include the 


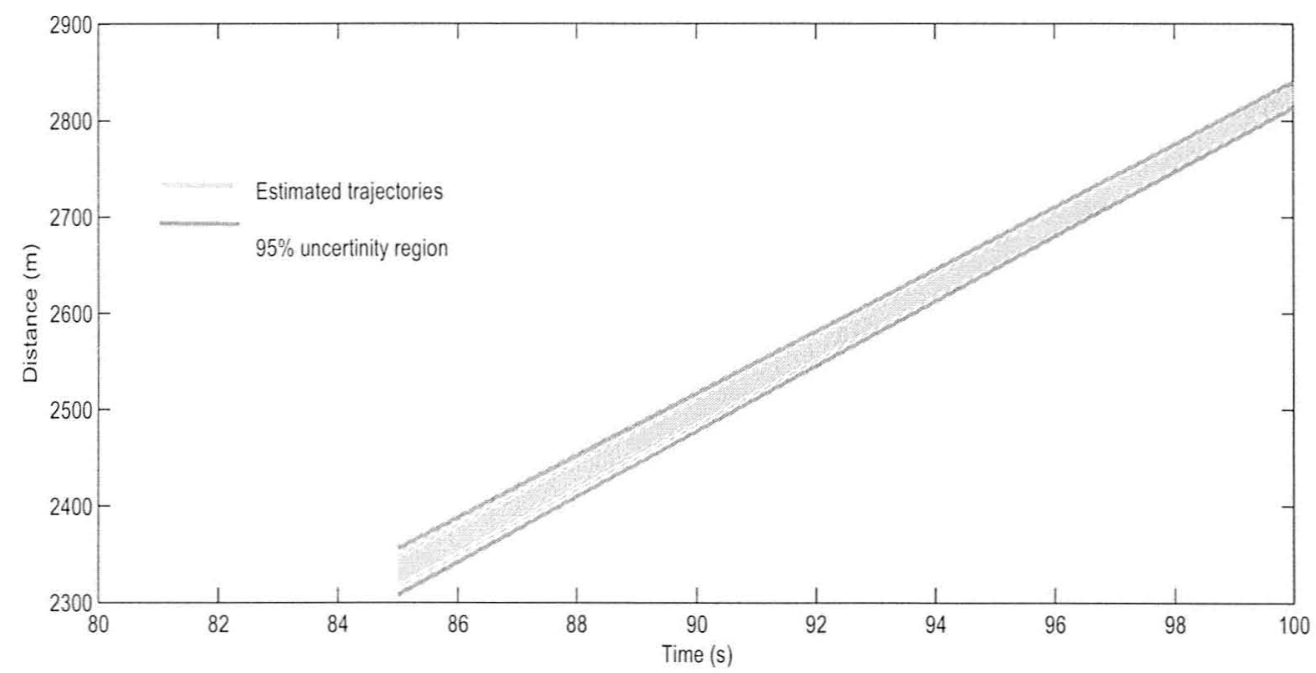

(a) At initial (CJML-PDA)

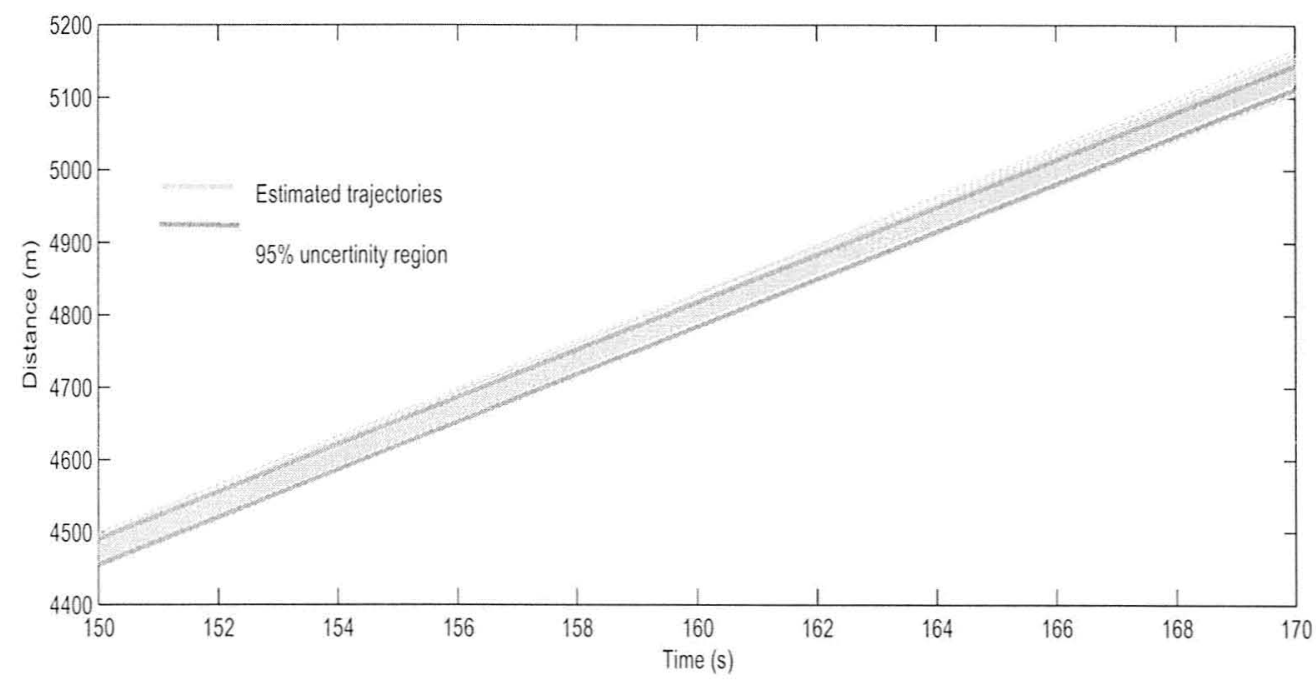

(b) At maintenance (JPDA)

Figure 5.2: Estimated trajectories of target 2 over 100 runs. 


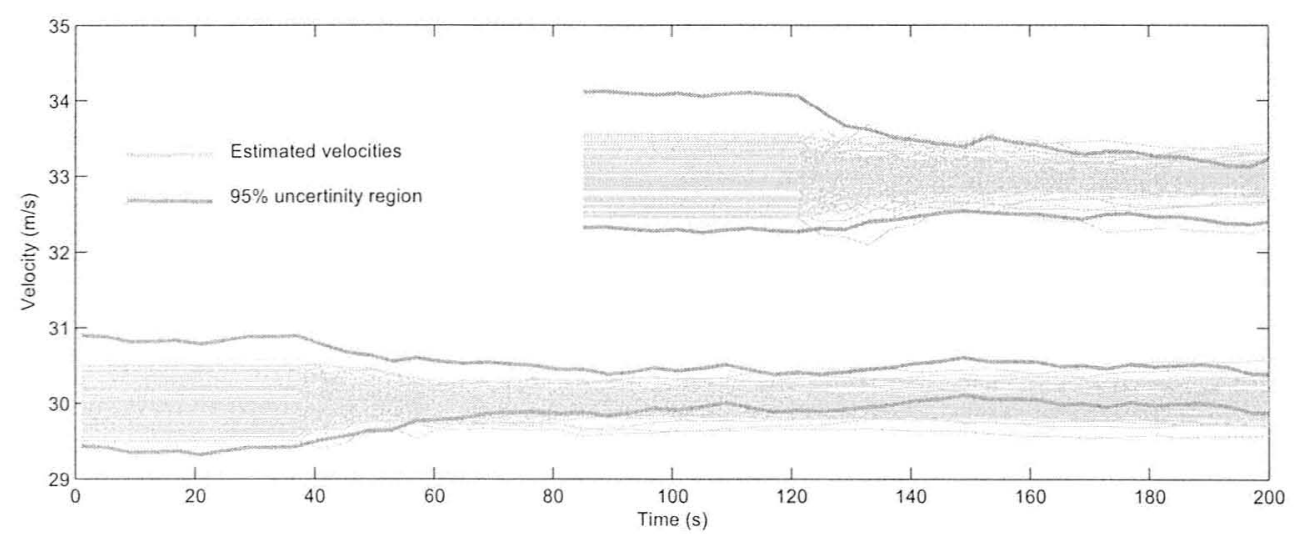

Figure 5.3: Estimated velocities of targets 1 and 2 over 100 runs.

corresponding Cramer-Rao Lower Bound that were described earlier. It is noticed that the RMSE of CJML-PDA position estimates are very close to the CRLB. Thus, it is clear that track initialization is well handled by CJML-PDA in heavy clutter and in the presence of a neighbouring targets. Initialized targets were maintained by the JPDA tracker, and it is clear that the estimated error are larger than the CRLB in some runs. The same was also observed for the RMSE velocity component. These results show that the mulitarget CJML-PDA and JPDA trackers perform well. Moreover, the detection component CJML-PDA handled the track detection successfully in high clutter and in the presence of neighbouring targets with the proposed information sharing technique between the modules. 


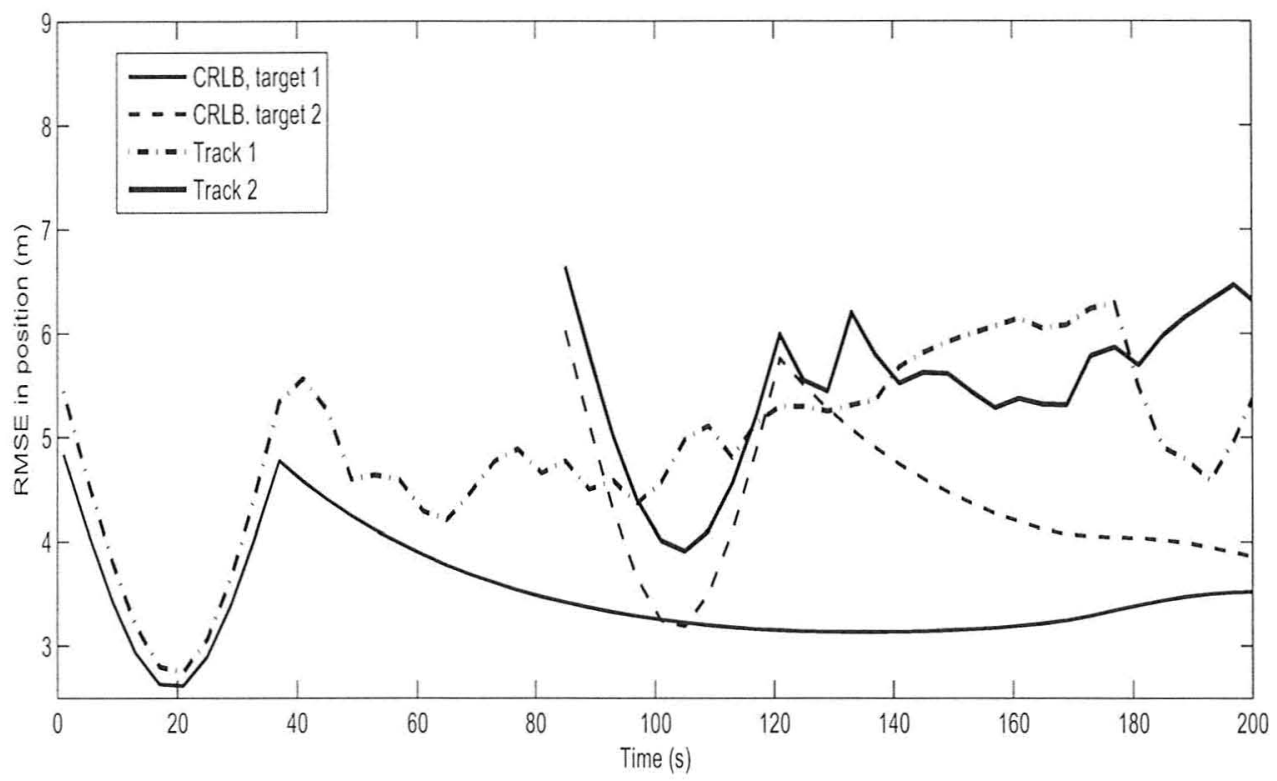

Figure 5.4: RMSE in position estimates of target states.

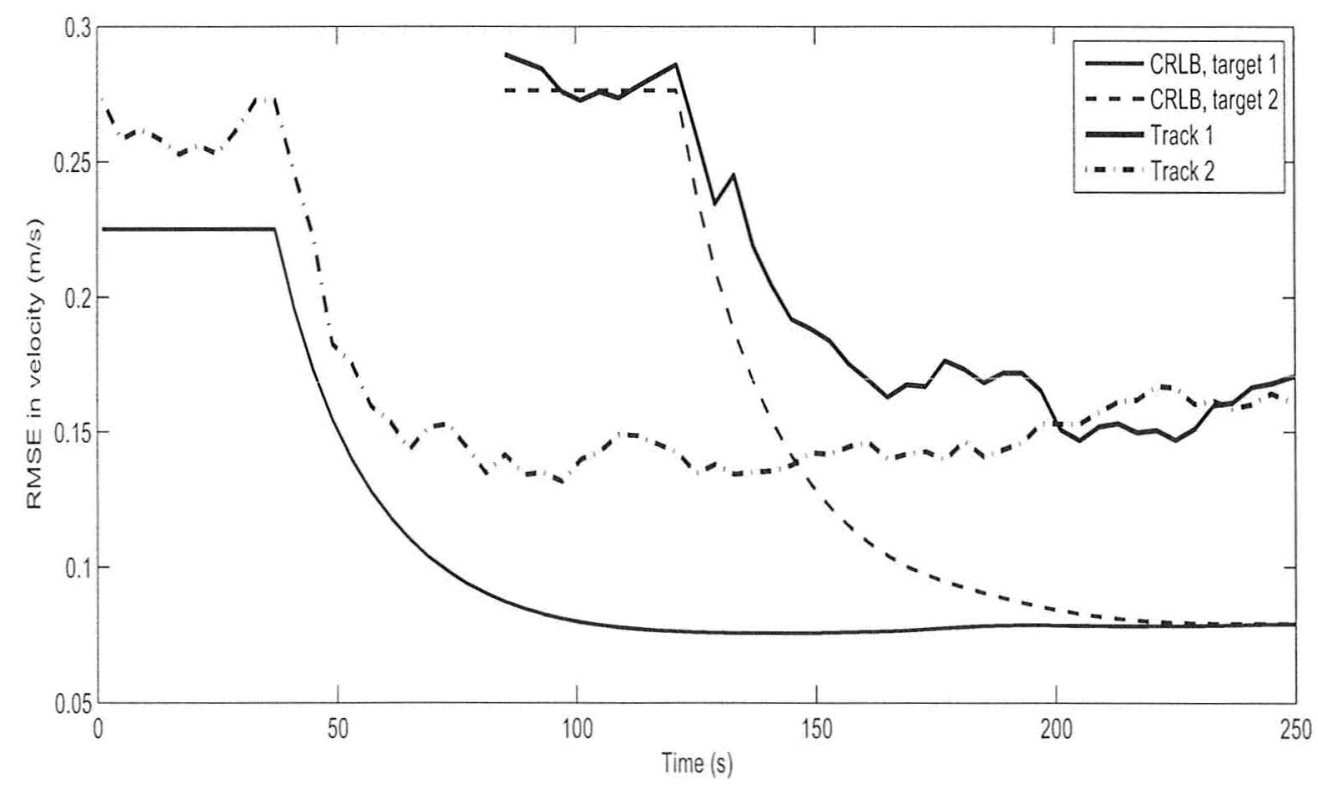

Figure 5.5: RMSE in velocity estimates of target states. 


\section{Chapter 6}

\section{Conclusions and Future Work}

\subsection{Conclusions}

In this thesis a new PDA based multitarget algorithm, called "Integrated CJML-PDA and JPDA tracker" was presented. Simultaneous track detection and maintenance were performed on two integrated modules CJML-PDA and JPDA, respectively, with sharing of information between the two tracking modules. The new CJML-PDA algorithm is a modified version of the JML-PDA technically that was used to handle track maintenance. Due to the lack of JML-PDA maintenance capability and higher computation complexity, CJML-PDA algorithm is formulated to handle track detection with the help of JPDA tracker. The thesis also included a real-time CJML-PDA JLLR optimization technique with a restricted directed subspace search (RDSS) that selectively maps measurements to the parameter space to provide restricted localized search among those points.

The tracker was tested on a simulated scenario consisting of two closely-spaced (crossing) targets with an angle-only sensor. The data also has a high number of 
false alarms. The result obtained from Monte Carlo simulations prove the capability of the proposed algorithm. It was found that the CJML-PDA RMS errors of both distance and velocity estimates are close to the CRLB. The algorithm handled the track detection and maintenance under heavy clutter and in the presence of neighbouring targets. This approach is not only restricted to angle-only problem as in the presented simulation, but also to any target and measurement space.

\subsection{Future Work}

Further work is required to evaluate the performance of the tracker with different types of targets as well as for different types of sensors. Additionally, the AI of measurements can also be added into the tracker. The AI is useful not only to validate measurements, but also in tracking. The strength of the CJML-PDA is track initialization presence of targets in heavy clutter. However, a track termination method is also needed for a complete tracker. The standard JPDA uses only a single scan of data. The JPDA could be extended to multiple scans. 


\section{Bibliography}

A. Rodningsdy, Y. Bar-Shalom, O. Hallingstad and J. Glattetre (June 2007). Multitarget tracking in the presence of wakes. 11th International Conference on Information Fusion, pages pp. $1-8$.

C. Jauffret and Y. Bar-Shalom (Nov 1990). Track formation with bearing and frequency measurement in clutter. IEEE Transactions on Aerospace and Electronic Systems, Vol. 26, pp. $999-1010$.

D. Lerro and Y. Bar-Shalom (Apr 1993). Interacting multiple model tracking with target amplitude feature. IEEE Transactions on Aerospace and Electronic Systems, Vol. 29, pp. $494-509$.

D. Lerro and Y. Bar-Shalom (Aug 2002). Interacting multiple model tracking with target amplitude feature. IEEE Transactions on Aerospace and Electronic Systems, Vol. 29, pp. $494-509$.

Goldberg, D. E. (1989). Genetic Algorithms in Search, Optimization, and Machine Learning. Addison-Wesley Boston, United States.

L. A. Johnston and V. Krishnamurthy (Aug 2002). Performance analysis of a dynamic 
programming track before detect algorithm. IEEE Transactions on Aerospace and Electronic Systems, Vol. 38, pp. $228-242$.

M. R. Chummun, Y. Bar-Shalom and T. Kirubarajan (Aug 2002). Adaptive earlydetection ML-PDA estimator for low observable target with electro-optical sensors. IEEE Transactions on Aerospace and Electronic Systems, Vol. 38, pp. $694-707$.

R. Tharmarasa, T. Kirubarajan, M. L. Hernandez and A. Sinha (April 2007). PCRLBbased multisensor array management for multitarget tracking. IEEE Transactions on Aerospace and Electronic Systems, Vol. 43, pp. 539 - 555.

T. Kirubarajan and Y. Bar-Shalom (Oct 1996). Low obsevable target motion analysis using amplitude information. IEEE Transactions on Aerospace and Electronic Systems, Vol. 32, pp. $1367-1384$.

T. Kirubarajan, Y. Bar-Shalom and D. Lerro (Jul 2001). Bearing-only target of maneuvering targets using a batch-recursive estimator. IEEE Transactions on Aerospace and Electronic Systems, Vol. 37, pp. 770 - 780.

W. Blanding, P. Willett and Y. Bar-Shalom (March 2007a). MLPDA: Advance and a multitarget approach. EURASIP Journal on Advances in Signal Processing, Vol. 2008, pp. 1-13.

W. Blanding, P. Willett and Y. Bar-Shalom (March 2007b). Multiple target tracking using maximum likelihood probabilistic data association. IEEE Aerospace Conference, pages pp. $1-12$.

W. R. Blanding, P. K. Willett, Y. Bar-Shalom and R. Lynch (April 2008). Direct 
subspace search ML-PDA with application to active sonar tracking. IEEE Transactions on Aerospace and Electronic Systems, Vol. 44, pp. 201-216.

X. R. Li and V. P. Jilkov (Oct 2003). Survey of maneuvering target tracking. Part I. Dynamic models. IEEE Transactions on Aerospace and Electronic Systems, Vol. 39, pp. $1333-1364$.

Y. Bar-Shalom and X. R. Li (1995). Multitarget-multisensor Tracking: Principles and Techniques. YBS Publications, United States.

Y. Bar-Shalom, F. Daum and J. Huang (Dec. 2009). The probabilistic data association filter. IEEE Control System Magazine, Vol. 29, pp. 82 - 100.

Y. Bar-Shalom, X. R. Li and T. Kirubarajan (2001). Estimation with Applications to Tracking and Navigation. Wiley-Interscience publications, Canada. 